\title{
Development of a Signal Recovery Algorithm for Overcoming PAPR in OFDMA Communication System
}

\author{
Mohamed S. El_Tokhy, Elsayed H. Ali * and H. Kasban \\ Engineering Department, Nuclear Research Center, Egyptian Atomic Energy Authority, Inshas, Egypt
}

\begin{tabular}{|c|c|}
\hline ARTICLE INFO & ABSTRACT \\
\hline Article history: & This paper focuses on overcoming the high Peak to Average Power Ratio challenging \\
\hline Received: $9^{\text {th }}$ Feb. 2021 & within Orthogonal Frequency Division Multiplexing Asynchronous (OFDMA) \\
\hline Accepted: $29^{\text {th }}$ July 2021 & communication system. This signal is modulated using differential phase-shift keying \\
\hline Keywords: & and quadrature amplitude modulation techniques. It is degraded under different \\
\hline OFDMA, & noise attacks. Signal recovery algorithms are supposed for overcoming the high peak \\
\hline Signal Transmission, & power problem. This issue is resolved through the detection of maximum peaks \\
\hline$H P A$, & within threshold peak values of the power amplifier output signal. Additionally, these \\
\hline$P A P R$ & peaks are identified depending on the extracted power density and order statistics \\
\hline$P D S$, & features. This system is trained using an artificial neural network under different \\
\hline HOS. & $\begin{array}{l}\text { channel impairments. Besides, the error rate of this network is computed in presence } \\
\text { of different channel fadings. Rayleigh fading imposes the best performance for the } \\
\text { transmitted OFDMA signal. Moreover, recognition of } 100 \% \text { is attained using the } \\
\text { high order statistics compared to the power density features in presence of noise } \\
\text { attacks. }\end{array}$ \\
\hline
\end{tabular}

\section{INTRODUCTION}

The Orthogonal Frequency Division Multiplexing Asynchronous (a state-of-the-art technology in optical communications) is now a hot spot for high-speed optical communications, especially in access networks and passive optical networks (PONs)[1,2]. This may be due to its high spectral efficiency. Thus, OFDMA is a promising high-capacity data transmission technique[3].OFDMA reduces the inter-symbol interference and the inter-channel interference from the received signal[4-8]. Hence, OFDMA is a widely applied technique for wireless communications. It is resistant to chromatic dispersion and polarization mode dispersion.[9, 10]. Therefore, OFDMA has become an important modulation scheme for several applications such as digital audio broadcasting, terrestrial digital video broadcasting, and wireless network communication. One advantage of OFDMA is that it turns a frequency selective channel into a flat fading channel for each subcarrier. In other words, OFDMA is a promising technology for eliminating the frequency selectivity of the wireless communication channels [11-13]. The number of people using multimedia communication systems has greatly increased. Systems must be evaluated in an environment that has a fast multipath fading channel. [14-17]. However, the benefit that OFDMA brings to wireless systems over single-carrier systems includes robustness to multipath fading[18-20]. Therefore, the BER estimation method for OFDMA transmissions based on the CWT is investigated.

In time-domain OFDMA, the mutual orthogonality among waveforms is ensured even if the carrier spectra may be overlapped [21]. Orthogonality is performed using FFT of the input stream because it is considered a simple modulation [20, 22]. Techniques that can be used for modulation may be; PSK, DPSK, QAM and quadrature phase-shift keying (QPSK). Since the OFDM 
subcarriers are orthogonal to one another and they overlap without intervening. However, OFDMA is a significant transmitter with high PAPR which causes poor system efficiency.[23, 24]. Thus, components within a system must be preferably linear over a broad spectrum.[13, 19, 25]. Consequently, one of the key advantages of adaptive modulation is that it can be performed inexpensively and can be achieved quickly. $[6,21,26]$.The high PAPR make a problem that resides at the transmitter of the power amplifiers. Since the amplifier gain is extremely saturated. The amplifier does not adjust its response depending on the input power. [19, 27, 28]. High PAPR decreases the fiber input capacity. As a consequence, the optical signal to noise ratio (SNR) can suffer upon transmission. [18, 23]. In addition, it limits the maximum transmission distance $[18,23,29,30]$. Besides, high PAPR can induce distortion in the digital-to-analog/analog-to-digital converter and cause non-linear fiber effects in the longhaul wavelength-division of multiplexing light wave systems. [9]. Several suggestions have been put forward ranging from filtering, clipping, coding, partial transmission sequences, and selected mapping to reduce the PAPR of OFDMA signals. Most of the recent works have focused on minimizing the PAPR of OFDMA signals to enhance their performance [23]. Therefore, a complete analysis for the OFDMA time waveform signal is introduced. This study is organized as follows. OFDMA Modulation Algorithm is presented at section 2. Section 3 illustrates the signal Analysis of Proposed OFDMA Signal Transmission. While section 4 gives the results and discussion. The conclusion is depicted at the final section of the manuscript.

\section{OFDMA Modulation Algorithm}

OFDMA is the number of many independent signals modulated in the same bandwidth with $\mathrm{N}$ sub-channels [14]. The entire bandwidth is sub-channeled and data symbols are transmitted in parallel. The effective data length is then extended to minimize the inter-symbol interference from a selective fading frequency channel. It is expected that the channels will adjust slowly. The recipient can thus estimate the instantaneous connection quality and accurately transmit the corresponding information to the sender. [31]. The optimal adaptation of the relation is therefore tested. The frequencyselective channel of these systems can be presumed that the transmission rate of the device varies very slowly. Moreover, the length of one or more packet transmissions can be approximated as quasi-statical [2]. An algorithm for OFDMA signal generation and transmission over channel fading environment is presented in Fig. (1). This algorithm depends on Discrete Sine Transform (DST) and HOS for reconstruction filter and upconverter steps.

The OFDMA considered the multipath distortion at the receiver. It provides high-speed streams on a nonreliable channel in an environment of fading. OFDMA eliminates the occurrence of inter-symbol interference and inter-channel interference Cyclic Prefix (CP). The advantage of $\mathrm{CP}$ is to bring about a transition from a linear convolution to a cyclic convolution. Therefore, the overall transferred function can be diagonalized by the use of an inverse Fast Fourier Transform (IFFT) at the transmitter and a Fast Fourier Transform (FFT) at the receiver end.

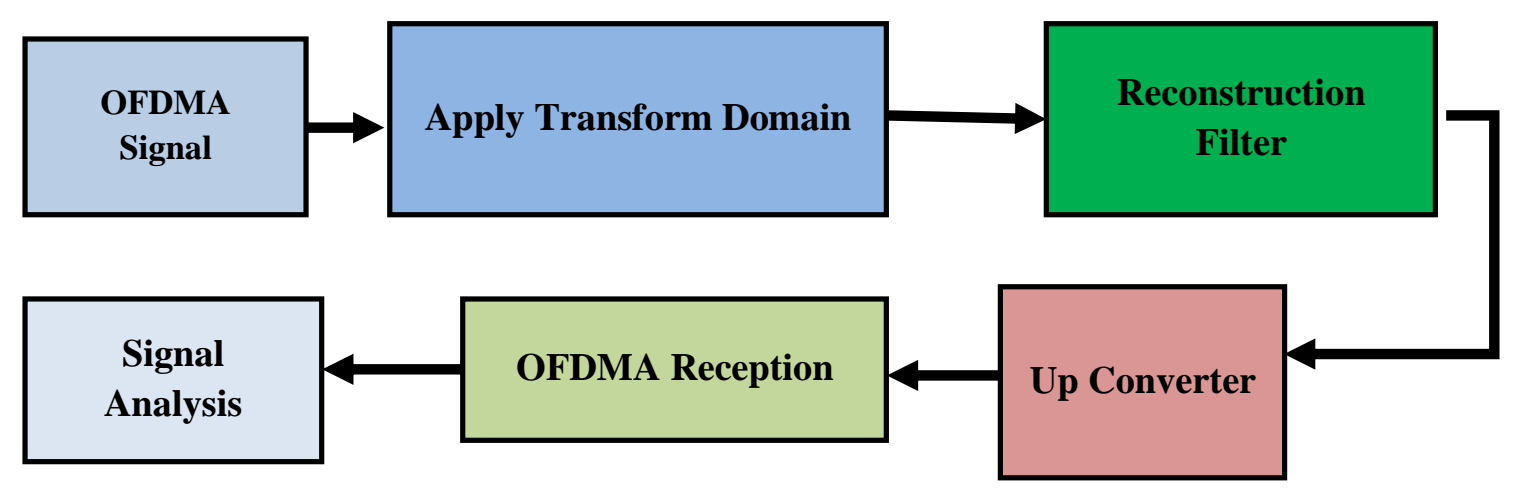

Fig. (1): OFDMA modulation algorithm based on DST and HOS 


\section{Signal analysis of proposed OFDMA signal transmission}

\subsection{Complex transform based OFDMA system}

The transformation of the wavelet takes place through a set of different scales of digital filters. This scaling procedure is carried out by sub-sampling by adjusting the signal resolution [6, 32, 33]. An algorithm for modulation purposes of OFDMA signal using CWT is investigated. This algorithm is proposed for multidimensional signal processing in previous works [5, 30, 34-36] is presented in Fig. (2). Moreover, different wavelet transform levels are considered.

One of the most important properties of the filters that can be performed to three-band filter banks is the phase linearity. The phase of the signals that are filtered using linear phase filters is not perturbed. Thus, the frequency components of the signal are shifted with the same values. The filter $\mathrm{h}(\mathrm{t})$ has the linear phase property which is in consistence with other investigations [35, 36]. The sampling frequency can be obtained by Nyquist's theorem in the ideal case. However, an anti-aliasing filter is applied before the sampling part in 0 non-ideal cases. Here, the sampling frequency can be obtained by [36]:

$$
f \geq f_{\mathrm{n}}+2 \mathrm{~B}_{\mathrm{tr}}
$$

Where fn and Btr denote the Nyquist frequency and the transient band of the anti-aliasing filter, respectively. The sampling rate is computed. Since the normalized bandwidth of the OFDMA signal is equal to the passband of the low-pass filter, the sampling frequency can be given by[36]:

$$
f_{s} \geq \frac{f_{n}}{2 B_{\text {low }}}
$$

Where Blow is the normalized pass-band of the lowpass filter.

The amount of sampling frequency increases with respect to the common sampling, this can be explained by[36]:

$$
R=\frac{f_{n} / 2 B_{l o w}}{f_{n}+2 B_{t r}}
$$

An orthogonal filter bank for OFDMA signal which consists of one real filter h0(n)and two complex conjugate filters $h_{c}(n)$ and $h_{c}^{*}(n)$ is studied. The complex filter is introduced in an earlier study [36]. These filters must satisfy the condition of the shift orthogonality to obtain the complete orthogonal transform. Moreover, the scaling and the wavelet functions must satisfy the condition of the shift orthogonality. Signal processing by inverse CWT is typically referred to as wavelet coefficient synthesizing. The reverse method is, however, the study of wavelet coefficients. [32].

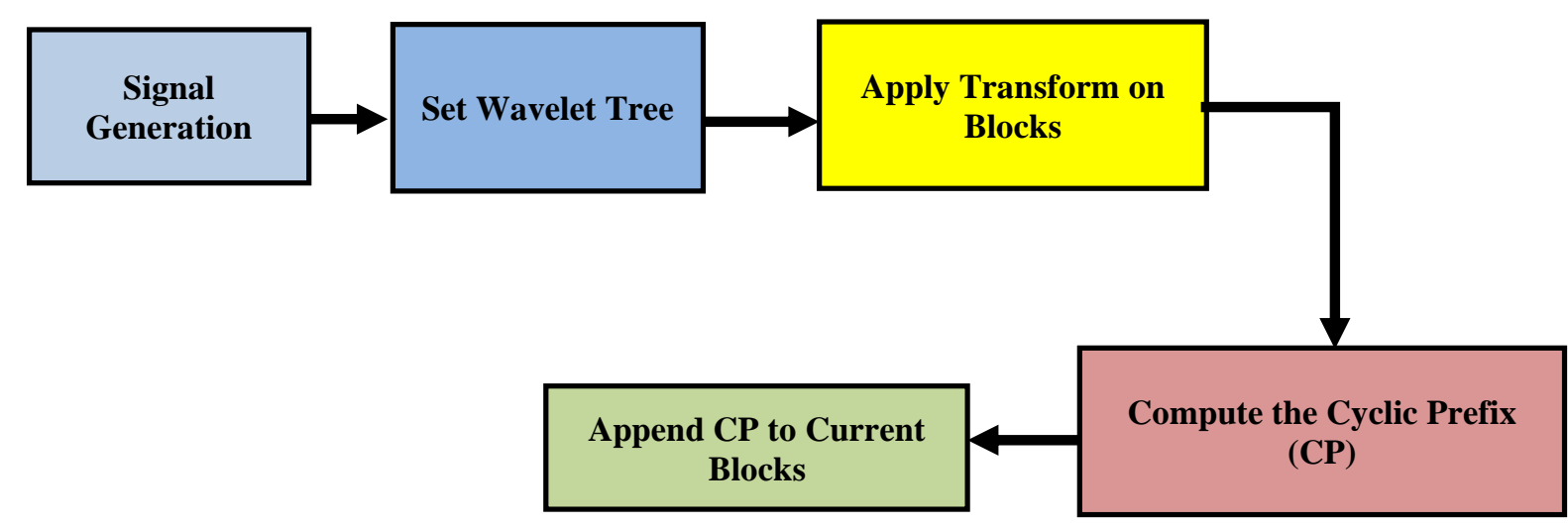

Fig. (2): The proposed OFDMA signal modulation algorithm at transmitter end using CWT 


\subsection{Effect of power amplifier on OFDMA signal}

However, the HPA is an essential device at the transmitter end of the OFDMA system for meeting the path loss and receiver sensitivity, it causes some distortion in the OFDMA signal due to its nonlinearity.[37].There are two types of commonly used HPAs, namely traveling wave tube amplifier and solidstate power amplifier.[37].On other hand, it is important to use a sophisticated and costly radio transmitter that has HPA. This is to overcome the very high PAPR in OFDMA based transmission systems. In addition, it can work in a very wide linear range. [38]. The effect of HPA on the transmitted OFDMA signal is described in the form of an algorithm presented in Fig. (3). One of the limitations of the HPA is the non-linear signal distortion. Thus, it may lead to a high adjacent interference and the performance degradation of the channel. These demands lead to complex and costly hardware systems. Thus, it is advisable to use the PAPR reduction techniques [38] in order to overcome the complex hardware design problem.

\subsection{High peak average power Reduction Algorithms}

The varying signal envelope with high peaks is the most noticed drawback of OFDM. It leads to a high PAPR for the transmitted signal [39]. This generates a broad magnitude of power and a highly effective dynamic range. The PAPR is the maximum peak to the average power of the sent OFDMA signals. [11]. It has a great effect on the RF amplifier efficiency[18].Therefore, the OFDMA transmitters require expensive linear amplifiers with a wide dynamic range [40].Moreover, the high PAPR requires HPA of a wide dynamic range at the transmitter [41]. The high peak power induces nonlinearity in the fiber channel for an optical communication system. Further, it causes the degradation of OFDMA system. The complex baseband signal can be given by $[24,37,40,42]$ :

$$
x(t)=\frac{1}{\sqrt{N}} \sum_{N-1}^{n=0} X_{n} e[2 j \pi n \Delta f t]
$$

Where $N, X n$, and $\Delta \mathrm{f}$ are the number of subcarriers, the data symbol at nth subcarrier, and the subcarrier frequency spacing, respectively. The high PAPR emerges from the summation in the above expression. [42].In other words, the high PAPR occurs due to the summation of many sub-carrier modulated signals. Moreover, the way of the sub-carrier phases can be aligned in the frequency domain. The transmitted signal PAPR was reported in previous works [10, 11, 40, 42]. The reduction techniques of PAPR are concerned with reducing the maximum values of the OFDMA signal power. Furthermore, the PAPR theoretical limit (in dB) depends on the number of subcarriers and it was referred to by Nadal et al. [22]:

$$
P A P R=10 \log _{10} N
$$

There are some techniques of reducing PAPR in OFDMA systems such as selected mapping and partial transmit sequence. However, these methods involve a high computational complexity [43].However, an algorithm for reduction of PAPR in OFDMA is introduced in Fig.( 4).This algorithm is evaluated in terms of statistical measurements.

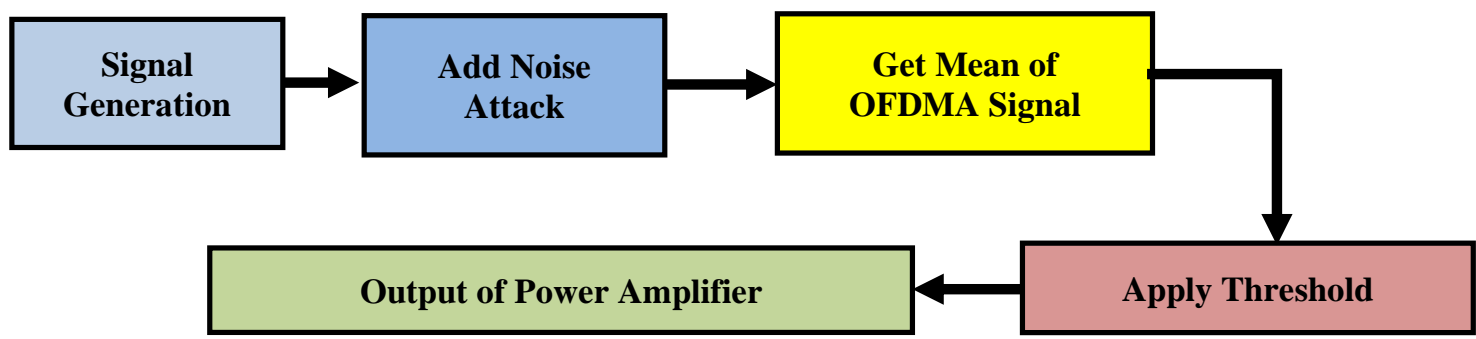

Fig. (3): An algorithm shows the impact of how OFDM is influenced by HPA

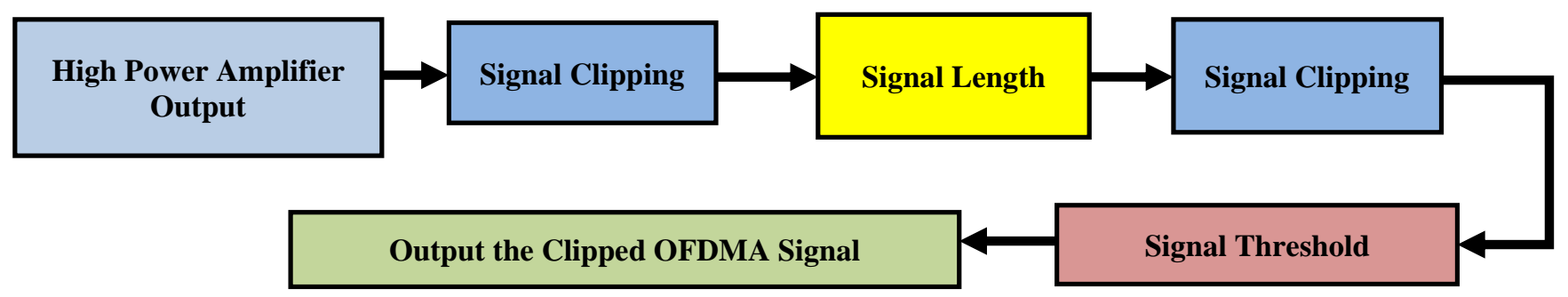

Fig. (4): The clipping algorithm for reduction of PAPR of OFDMA signal 


\subsection{OFDMA Signal recovery algorithm}

An algorithm presented to reduce the PAPR of the modulated OFDMA signal is shown in Fig. (5). This algorithm depends on the detection of maximum peaks of the HPA output signal. Moreover, the detected peaks depend on the implemented peak routine. Then, this peak is reduced to be within upper and lower threshold peaks as well as the detected peak above or below these limits. The accuracy of this algorithm is measured by comparison with the general clipping method and statistical merits.

\subsection{OFDMA signal recognition under channel impairments}

AWGN noise influence and other channel degradations are considered. Furthermore, there is a multipath propagation for verification of the properties of the recognizer $[6,7,20,29]$.Realistic channel modeling is essential for accurate wireless network simulation. The recognition of the OFDMA signal is affected by these impairments. Consequently, two different algorithms are used for OFDMA signal recognition. These are based on PDS and high-order statistics HOS.

\subsubsection{Power spectrum of OFDMA signal recognition}

One of the must-have tools in digital signal processing is PDS estimation, a method for estimating the discretetime deterministic and stochastic process's power spectrum [6, 44, 45]. The Fourier transform does not exist for infinite energy signals. Therefore, their PDS can be used for characterizing their spectrum. There are different algorithms that can be used for PDS estimation. There are three basic types of statistical methods: non-parametric, parametric, and eigenvalue analysis $[6,46,47]$.

The nonparametric PDS implies that the estimation methods do not presume that the data was generated in a specific way. There are nonparametric methods that can be used for PDS estimates, such as Periodogram, Welch, and Blackman using Tukey methods [48]. The key downside of nonparametric spectral estimation techniques is the effect of off-side lobe leakage due to finite data sets [49].The periodogram can be given by[50]:

$$
P^{\text {Per }}(k)=\frac{1}{N_{s}}\left|\sum_{n=0}^{N_{s}-1} E(n) \exp [-j 2 \pi k n]\right|^{2}
$$

Where $\mathrm{E}(\mathrm{n})$ denote the discrete signal and Ns is signal length. Two adaptations have been implemented using Welch and Blackman with Tukey methods to address periodogram limitations. In addition, three methods are used to estimate the PDS using the own analysis; the own vector method, the Multiple SIgnal Classification (MUSIC) method, and the multi-taper method. [46]. These methods have been previously described in detail [51]. Therefore, an algorithm for OFDMA signal recognition based on PDS is proposed in Fig. (6). The PDS of OFDMA signal recognition has two different phases. These phases are the training and testing phases.

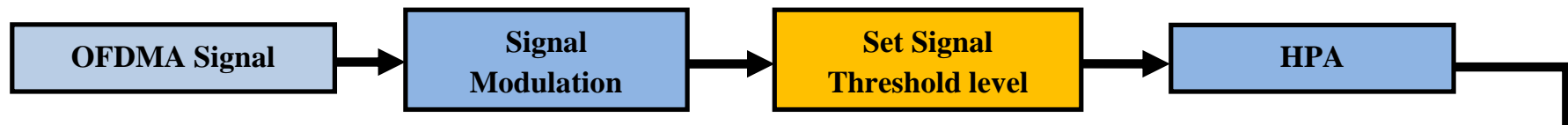

Get Reconstructed (actual) OFDMA Signal

Apply Peak Routine

Fig. (5): OFDMA signal recovery algorithm based on maximum detected peaks method

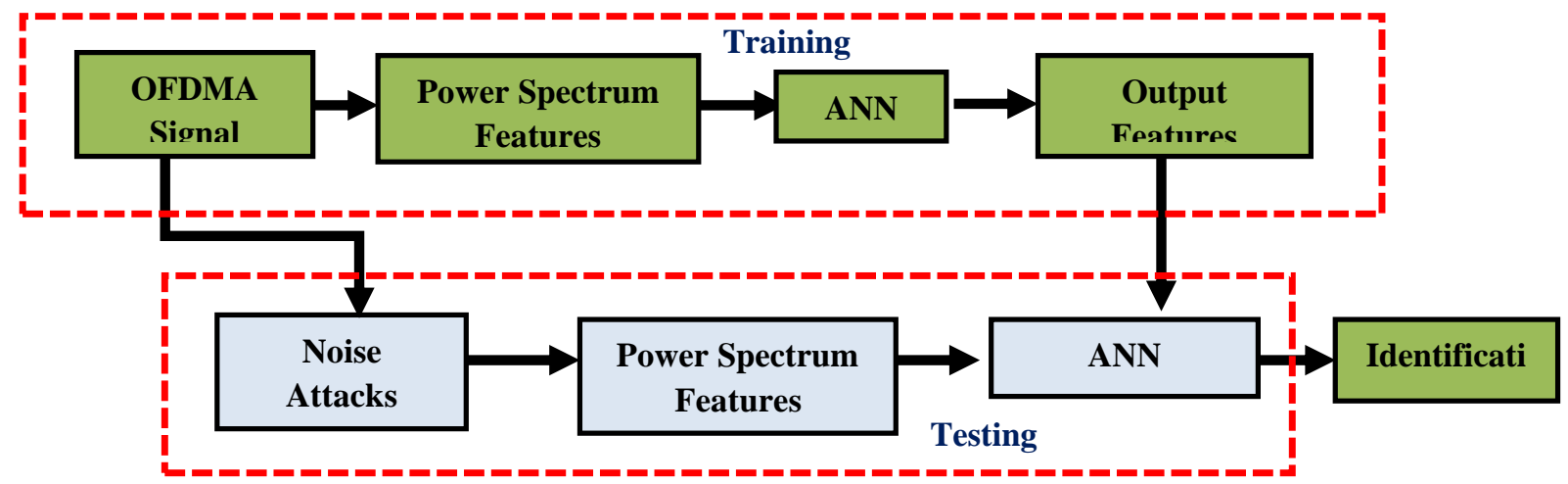

Fig. (6): OFDMA signal identification algorithm based on PDS 
A database of 20 OFDMA signals is used in the training phase. The PDS of these signals were calculated using various methods such as non-parametric (Periodogram and Welch), parametric (Yule-Walker, Burg and Covariance), and self-analysis (eigenvector, MUSIC, and multi-taper) methods. The features are derived from the OFDMA PDS signal. These features are used to train the neural network. The features are removed from each PDS of the signal after they have been corrupted during the test process. The OFDMA signal is distorted by the addition of numerous noises. These sounds are white Gaussian noise, Rayleigh Noise, Rician Noise, white Gaussian noise and Rayleigh, white Gaussian noise with Rician, Rayleigh with Rician, white Gaussian noise with Rayleigh and Rician, and Salt pepper noise. Finally, a feature matching step is performed to determine whether or not these features belong to the OFDMA signal or to the artificial neural network (ANN) signal.

\subsubsection{Statistical power of OFDMA Signal Recognition}

The application of HOS to practical problems is demonstrated[52].Thus, HOS for the recognition of OFDMA signal processing is proposed. The Bi-spectrum is capable of detecting and quantifying the phase coupling. Two approaches have been used for Bi-spectrum estimation. These are the traditional and parametric approaches focused on Auto-Regressive (AR), Moving Average (MA) and Auto-Regressive Moving Average (ARMA) models. They can be divided into direct and indirect groups. However, peak to average power ratio is computed, these approximations have limitations on the statistical uncertainty of the calculations and the time of the machine. Memory has serious issues with its implementation. Bi-spectrum is a two-frequency function and is truly valued and nonnegative in comparison to the power spectrum.[53]. $\mathrm{Bi}$-spectrum is complex-valued for a real-valued process, symmetry properties of cumulants carry over to symmetry properties of poly-spectra. The spectrum of power is as symmetrical as $\mathrm{S} 2 \mathrm{x}(\mathrm{f})=\mathrm{S} 2 \mathrm{x}(-\mathrm{f})$. The Bi-spectrum symmetry properties are previously reported [36]. Parametric estimators are often useful. Moreover, the underlying physics of the problem suggests a parametric model. If $x(n)$ depends on a finite set of parameters, $\theta$, then all of its figures are expressed in terms of $\theta$. The PDS parametric estimations are derived from the first estimation of $\theta$ and the evaluation of $\operatorname{Pxx}(f \mid \theta)$. A type of time series analysis is the ARMA model as given by Hosseini et al.[36]. The transfer function does not retain any phase information, and several methods have been developed to try to acquire it.

$$
\mathrm{H}(\mathrm{z})=\mathrm{B}(\mathrm{z}) / \mathrm{A}(\mathrm{z})
$$

The fundamental basis of cross Bi-spectrum is a Fourier transform of the third-order cumulant series [36]. Therefore, the cross bi-spectrum was previously defined [54]. An algorithm describing the extraction of characteristics from statistics of a high order is therefore investigated as shown in Fig. (7).

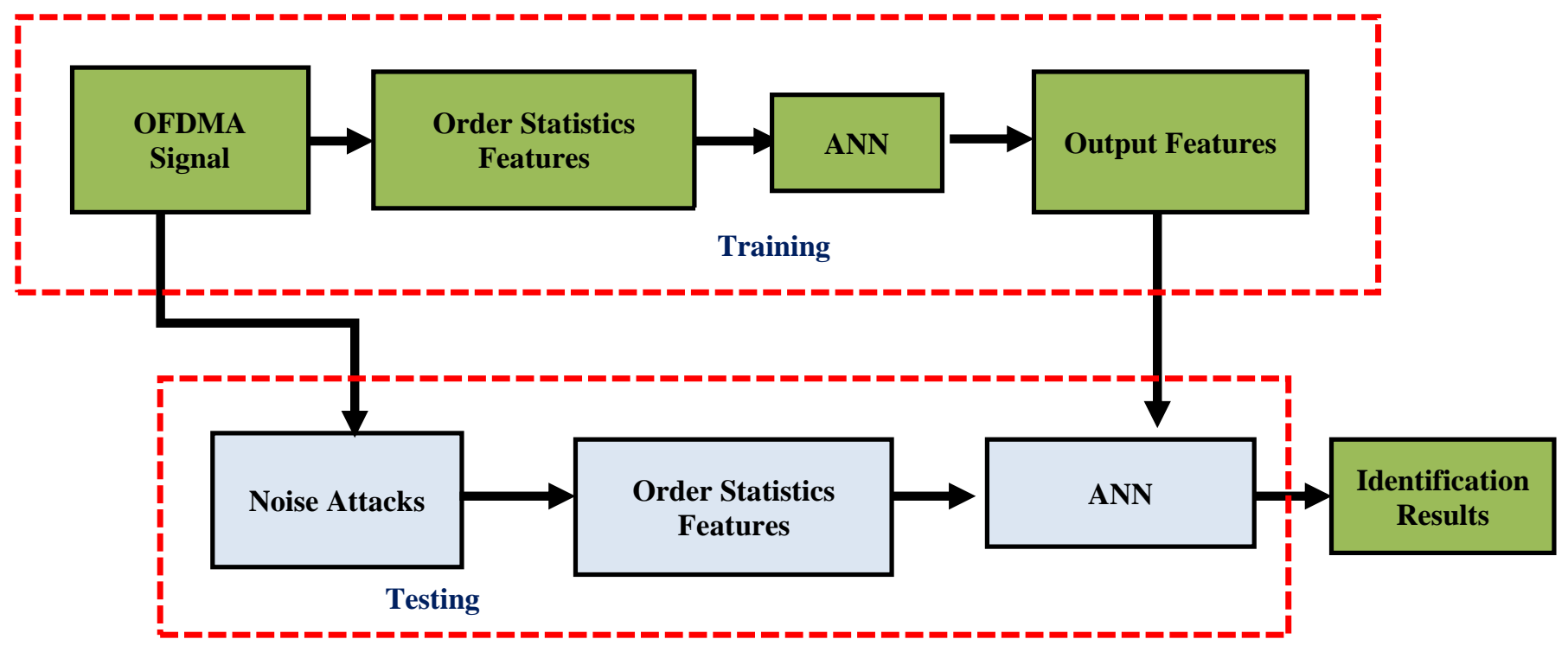

Fig. (7): OFDMA signal identification algorithm based on HOS 


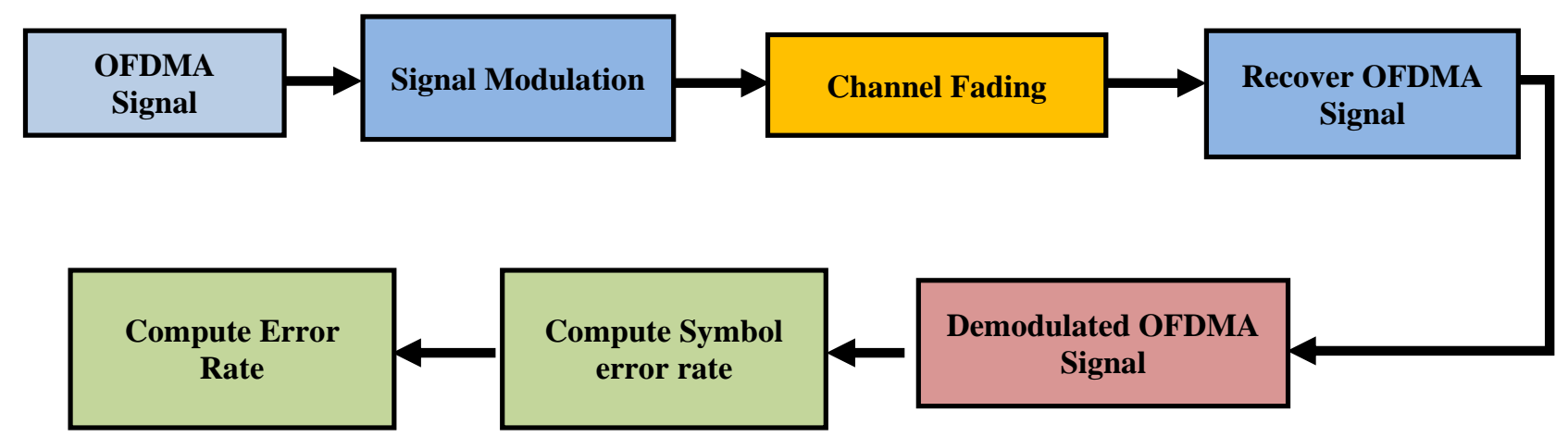

Fig. (8): The algorithm shows the effect of the different channels fading to the received OFDMA signal

\subsection{BER under channel fading algorithm}

It is both simple and less complicated to implement the clipping technique. However, the cutting processes cause the OFDMA signal to distort. BER is also improved [4, $8,43,55-57]$.BER is the ratio of the error number to the number of bits indicated. By counting the numbers of errors at the receiver with enough symbols registered, BER can be calculated directly. A precise and highly efficient BER estimate is therefore preferred to be used to understand, design, and optimize an OFDMA system $[5,12,30]$. Consequently, an algorithm that shows the channel fading effect on the modulated OFDMA signal is shown in Fig. (8). The BER results are derived as a function of the average symbol energy to noise spectral density (E0/N0) [58].The SNR is an indicator that can be used for evaluating the communication system quality[21, 31, 59, 60].

\section{RESULTS AND DISCUSSION}

\subsection{OFDMA signal recovery after HPA}

The influence of HPA on OFDMA Signal is declared. Therefore, the recovery of the original OFDMA signal is necessary. On the other hand, the signal amplitude depends on the type of modulation technique. Moreover, the OFDMA signal amplitude for PSK is higher than QAM. The effect of the modulation technique on the HPA output is computed by statistical comparison between PSK and QAM modulation techniques as depicted in Table (1). It is shown that the amplitude of the OFDMA signal increases with HPA. Thus, the OFDMA signal is changed after HPA.
Table (1): Statistical measurement for the effect of HPA based on CWT using both QAM and PSK modulation techniques

\begin{tabular}{ccc}
\hline $\begin{array}{c}\text { Statistical } \\
\text { Measurements }\end{array}$ & $\begin{array}{c}\text { OFDMA } \\
\text { Modulation by } \\
\text { QAM }\end{array}$ & $\begin{array}{c}\text { OFDMA } \\
\text { Modulation by } \\
\text { PSK }\end{array}$ \\
\hline PSNR & 55.182 & 54.8651 \\
MSE & 0.19719 & 0.21211 \\
Entropy & 2.5543 & 2.5161 \\
MAE & 0.16922 & 0.16313 \\
PCC & 31270.4294 & 31353.6841 \\
Error Rate & 0.3443 & 0.3144 \\
\hline
\end{tabular}

The OFDMA signal and output of HPA using the PSK modulation technique are shown in Fig. 9(a and b), respectively. The peak to average power ratio is computed and equal to $28.5122 \mathrm{~dB}$ for QAM. In addition, the OFDMA signal recovery using the PSK modulation technique is depicted in Fig. 9(c). However, it is equal to $26.8052 \mathrm{~dB}$ for PSK. The statistical evaluation of OFDMA signal recovery using QAM and PSK is shown in Table (2).

On the other hand, another OFDMA signal recovery method based on localizing the maximum peaks are introduced. This method finds the local maxima in the OFDMA signal at the output of OFDM. Moreover, this method has the advantage of determining the number of passes of the small running average filter to reject small peaks. The evaluation of the recovered OFDMA signal from HPA output is determined by statistical evaluation as shown in Table (2). The obtained results confirm that OFDMA signal recovery by maximum peak detected using QAM has yielded better results in terms of mean absolute error and BER than other approaches. In other words, it achieves a higher PSNR than other approaches. The proposed peak average power ratio algorithms are validated through a comparison with those reported in earlier studies $[13,24]$. 

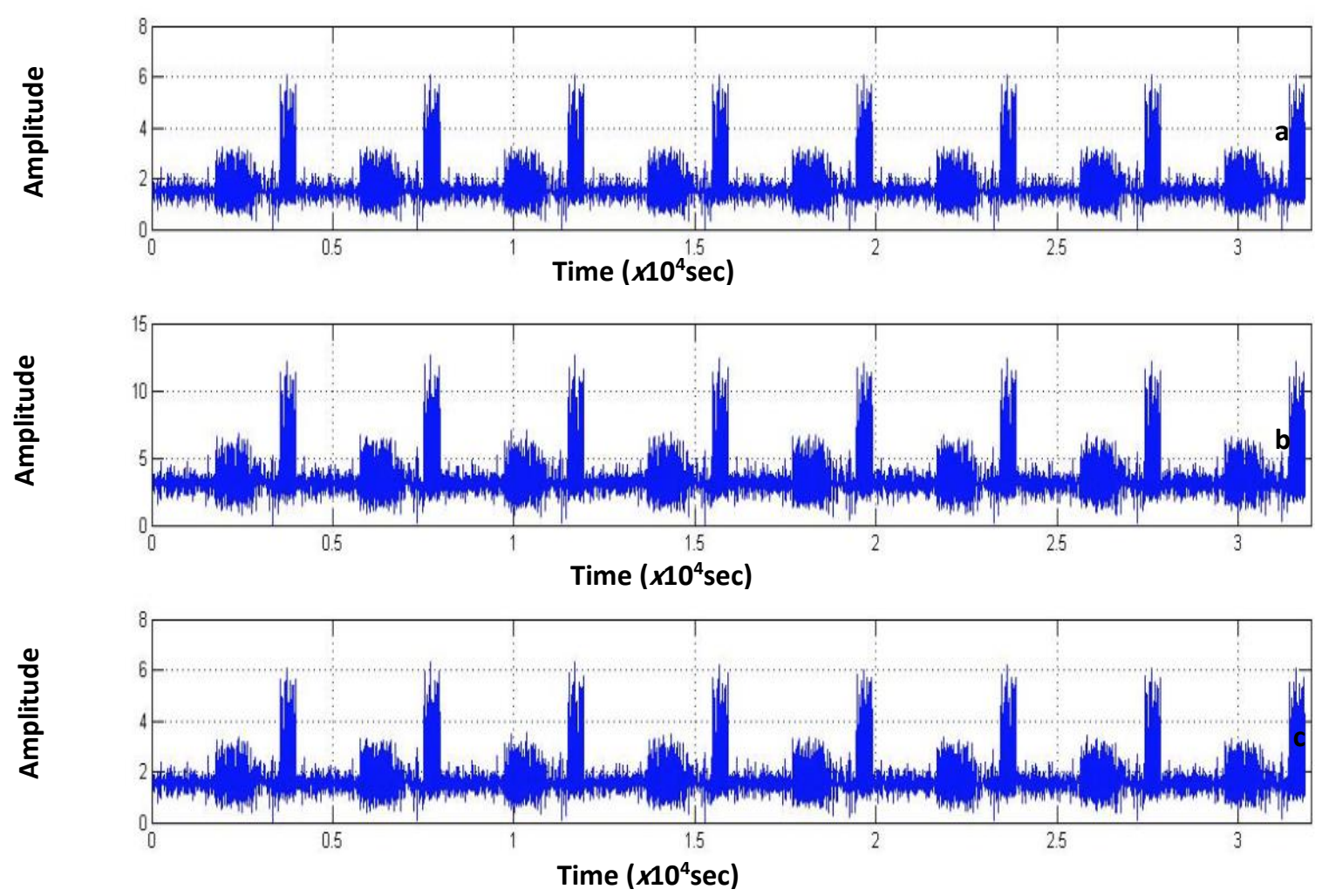

Fig. (9): The OFDMA signal recovery using PSK under White Gaussian noise fading effect

a) OFDMA signal b) OFDMA signal at output of HPA c) Clipped signal at output of HPA

Table (2): Statistical measurement for OFDMA signal recovery by clipping and maximum peak methods based on CWT using both QAM and PSK modulation techniques

\begin{tabular}{lcccc}
\hline $\begin{array}{c}\text { Statistical } \\
\text { Measurements }\end{array}$ & $\begin{array}{c}\text { OFDMA Signal } \\
\text { Recovery by QAM } \\
\text { Using Clipping }\end{array}$ & $\begin{array}{c}\text { OFDMA Signal } \\
\text { Recovery by PSK } \\
\text { Using Clipping }\end{array}$ & $\begin{array}{c}\text { OFDMA signal } \\
\text { Recovery by QAM } \\
\text { using Max Peaks }\end{array}$ & $\begin{array}{c}\text { OFDMA Rignal } \\
\text { Recovery by PSK } \\
\text { using Max Peaks }\end{array}$ \\
\hline PSNR & 44.95 & 44.26 & 44.50 & 43.49 \\
MSE & 2.07 & 2.43 & 2.30 & 2.90 \\
Entropy & 0.53 & 0.36 & 2.84 & 2.84 \\
MAE & 1.43 & 1.55 & 0.22 & 0.22 \\
PCC & 31298.42 & 31300.92 & 3898.66 & 4002.58 \\
BER & 1 & 1 & 0.02 & 0.03 \\
\hline
\end{tabular}

The effect of different fading channels on the transmitted OFDMA signal is depicted in Fig. (10). These communication fading effects are Rician, White Gaussian, Rayligh with Rician, complex multipath, and White Gaussian with Rayligh channel degradations. From this Figure, there is a variation for
BER observed at different values of SNR. It is noted that White Gaussian noise achieves the smallest BER for all values of SNR. However, the Rayligh with Rician channel noise introduces the highest BER. However, the BER dropped at a higher SNR for all types of fading channels. 


\subsection{OFDMA signal recognition}

Recently, there have been several developments in the algorithms where they can withstand fading channels, both in time and space. OFDMA signal recognition is based on two separate extracted function methodologies. These are the power density spectrum and the statistics of a high order. Multiple OFDMA signals have been considered for neural network training. With one of the underlined methods, the characteristics of the OFDMA signal were extracted from the original signal and their PDSs were estimated. The authors used the $1 \mathrm{kHz}$ sampling frequency and the 2000 points FFT filter length for all estimation methods. 3 is the order of the AR model. For the multi-taper form, the time-bandwidth product is 3. Moreover, identical features to those used in the training have been extracted from the degraded OFDMA signal in the testing process. For ANN training, on the other hand, 20 OFDMA signals are used. For matching purposes, 20 OFDMA signals are also fed to the neural network. To assess the efficiency of the proposed solution in the presence of noise, the tested signals have been degraded by various forms of noise. Gaussian, Rayleigh, Rician, Gaussian and Rayleigh, Gaussian and Rician, Rayleigh and Rician, Gaussian with Rayleigh and Rician and Salt Pepper are the degradations to be considered. Even, with signal deformation, these effects are considered.

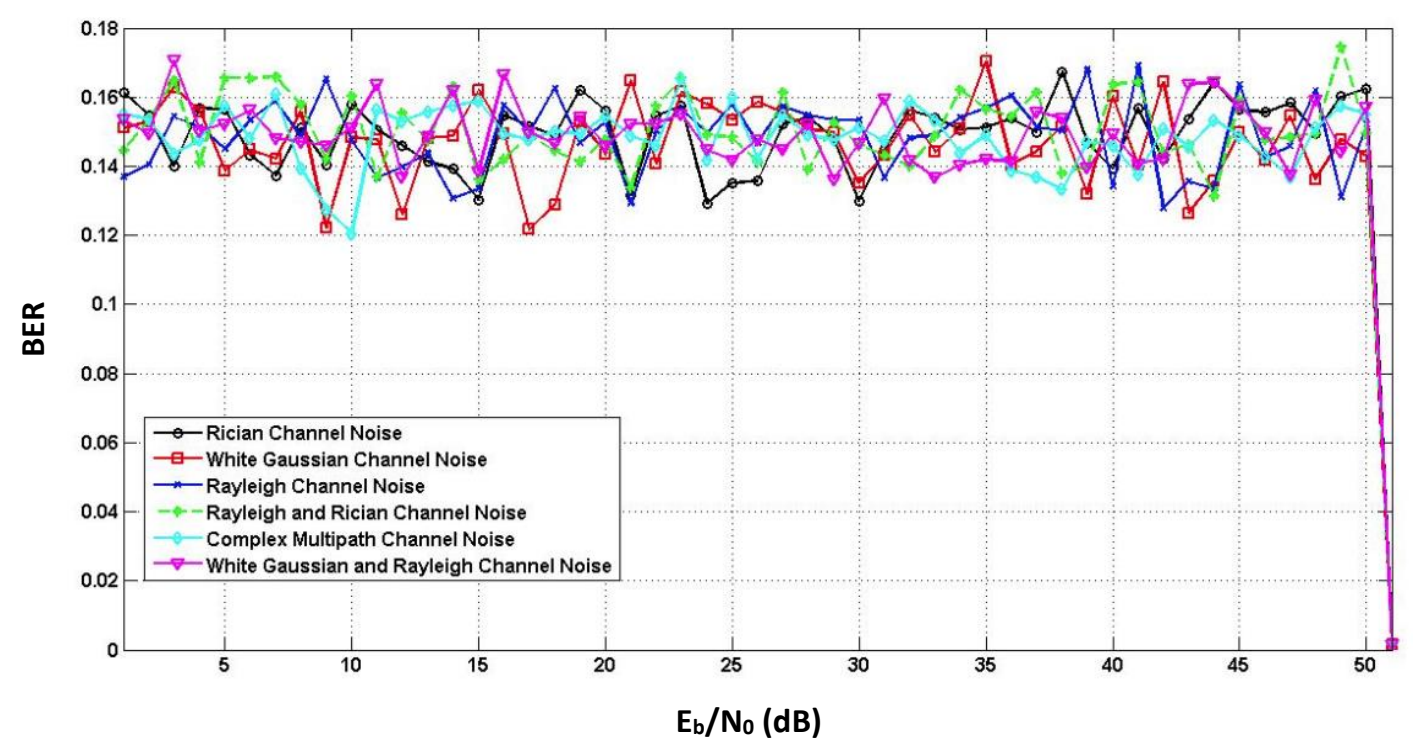

Fig. (10): The bit error rate against signal to noise ratio under different channel noise fading effects

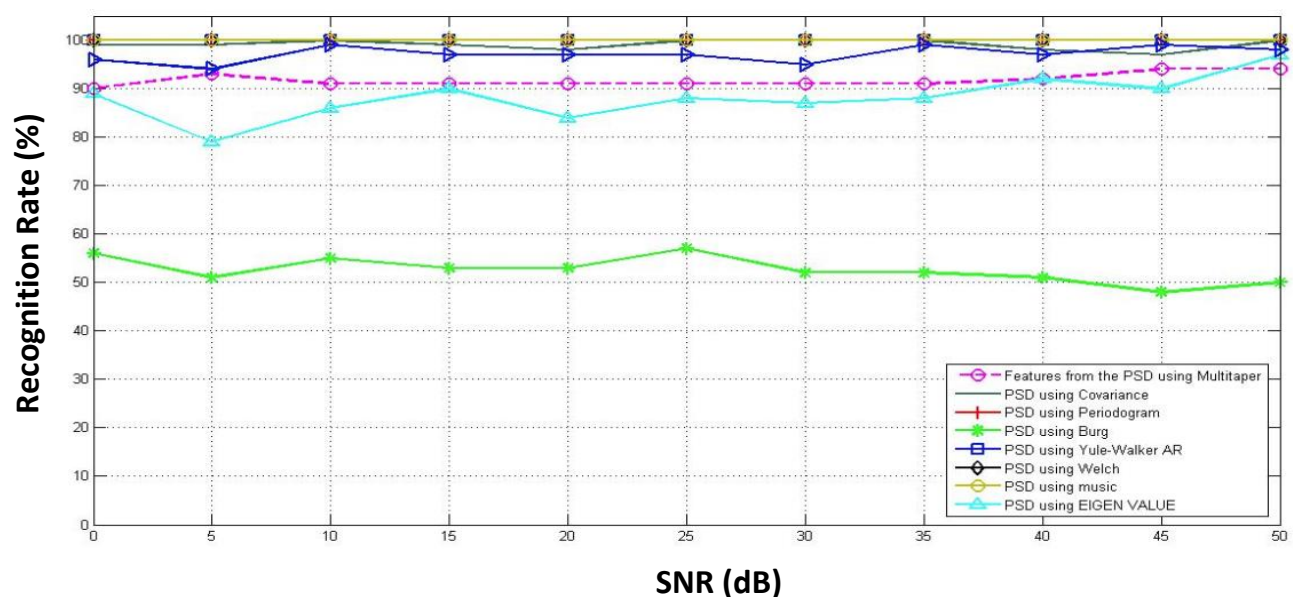

Fig. (11): Recognition rate versus SNR for parametric, non-parametric and Eigen vector analysis with Gaussian degradation 


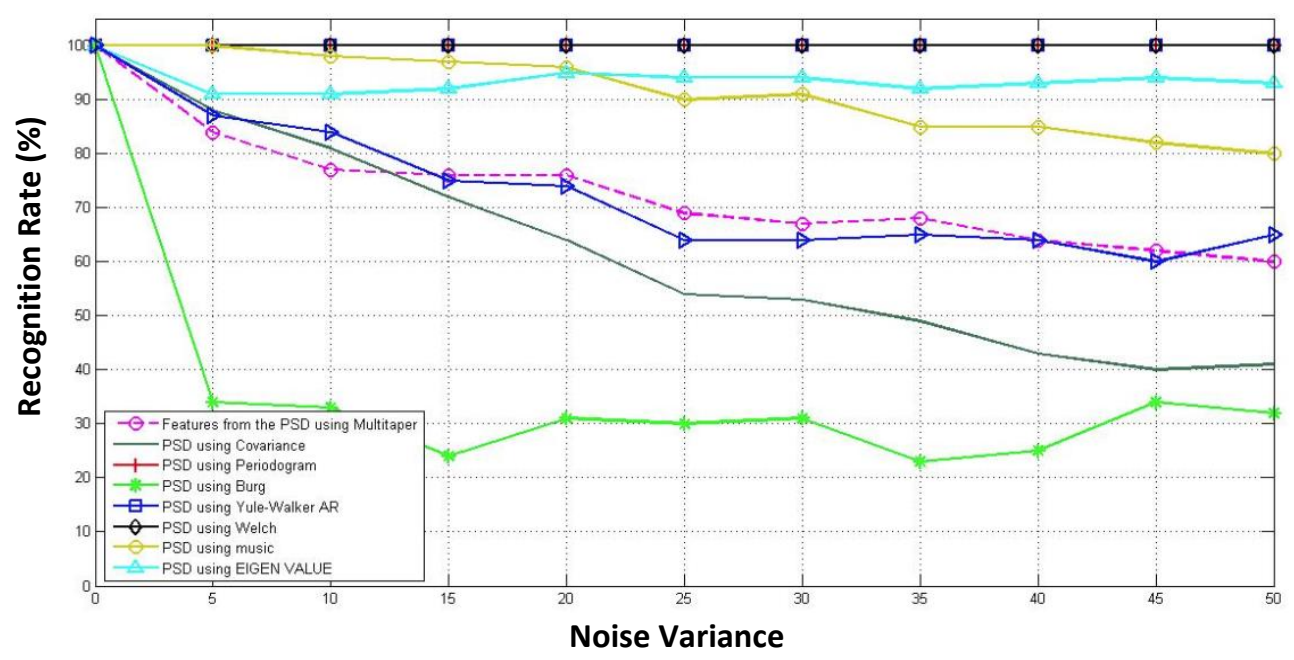

Fig. (12): Recognition rate versus noise variance for parametric, non-parametric and Eigen vector analysis with Rayleigh degradation

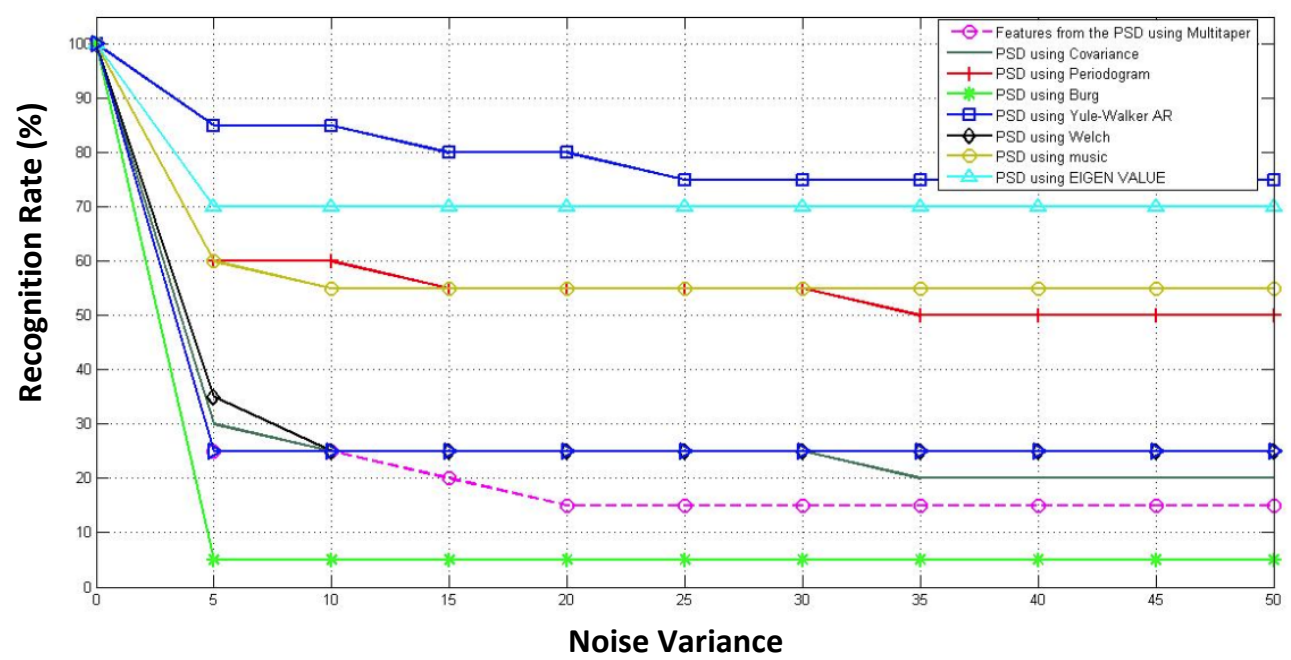

Fig. (13): Recognition rate versus noise variance for parametric, non-parametric and Eigen vector analysis with Rician degradation

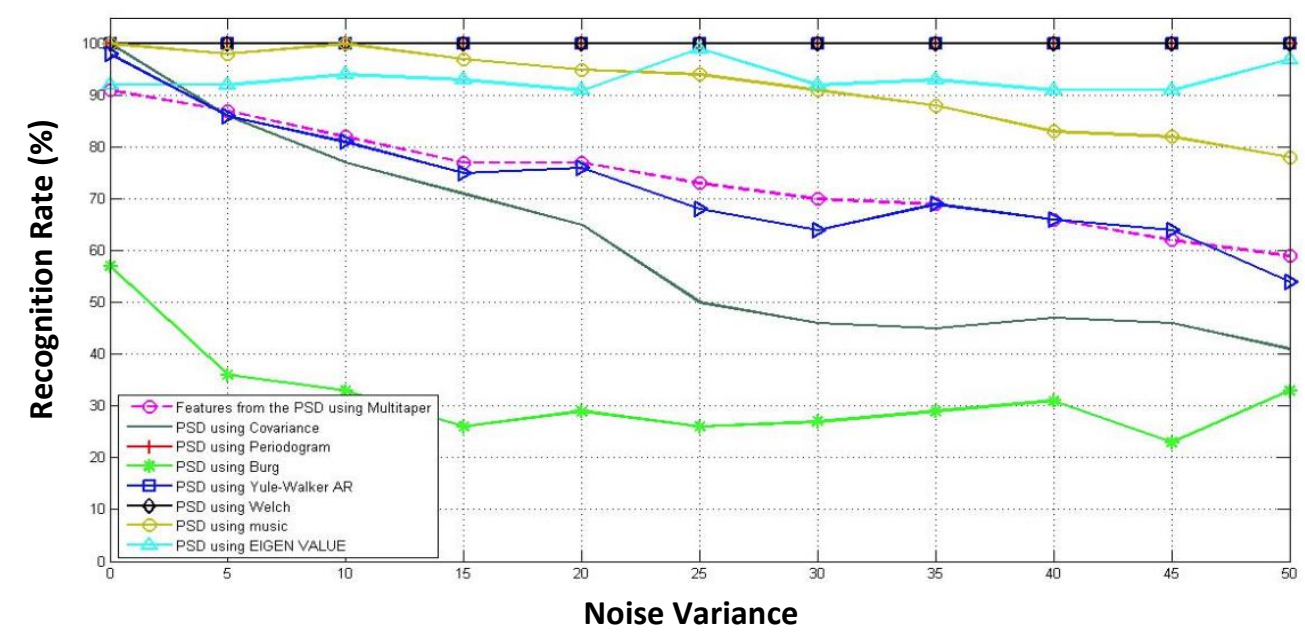

Fig. (14): Rate of recognition versus noise variance with Gaussian and Rayleigh degradation for parametric, non-parametric and Eigen vector analysis 


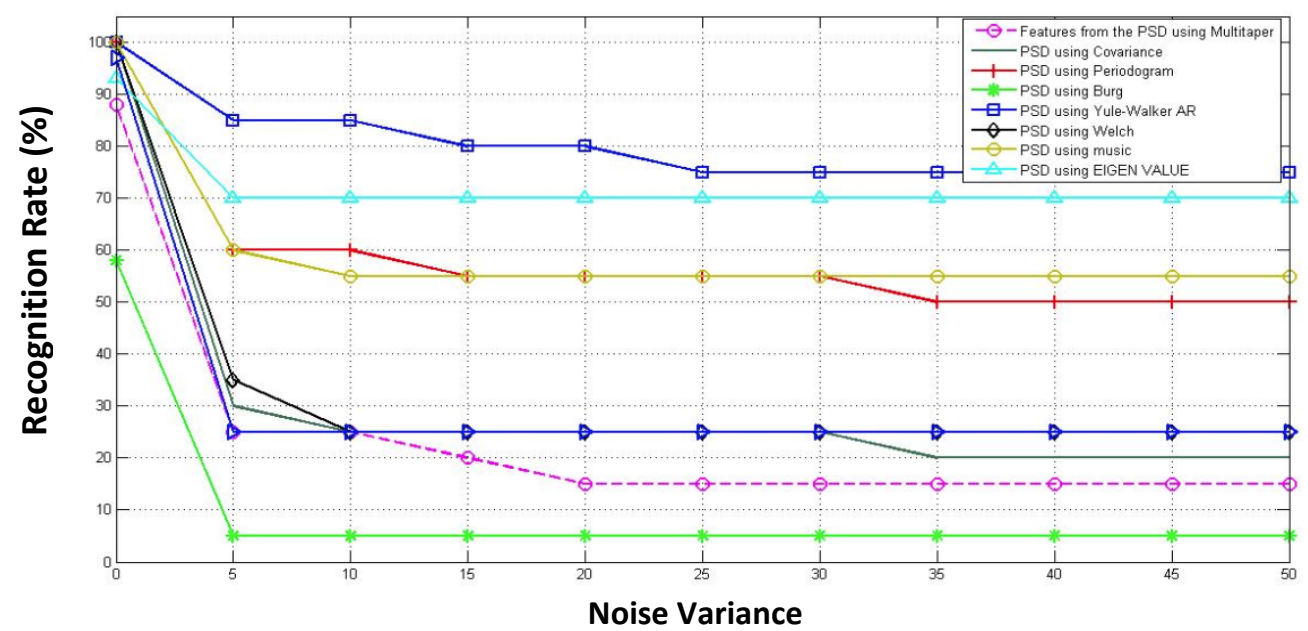

Fig. (15): Recognition rate versus noise variance with Gaussian and Rician degradation for parametric, non-parametric, and Eigen vector analysis

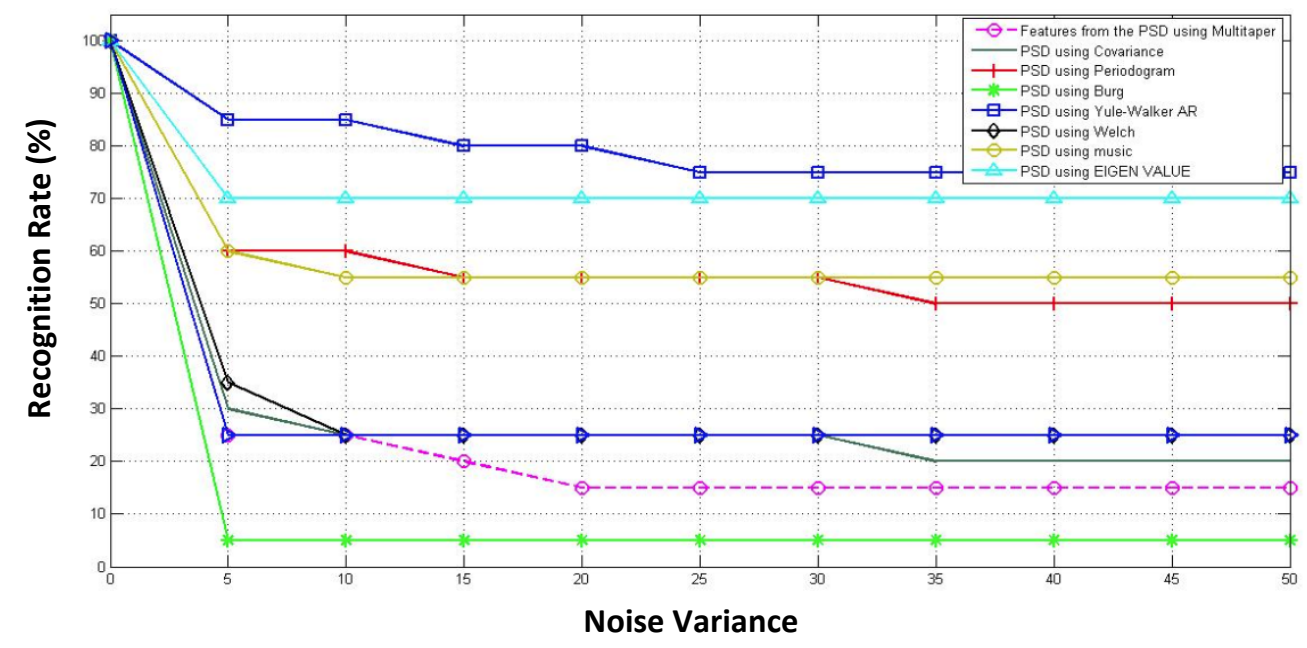

Fig. (16): Recognition rate versus noise variance for parametric, non-parametric and Eigen vector analysis with Rayleigh and Rician degradation

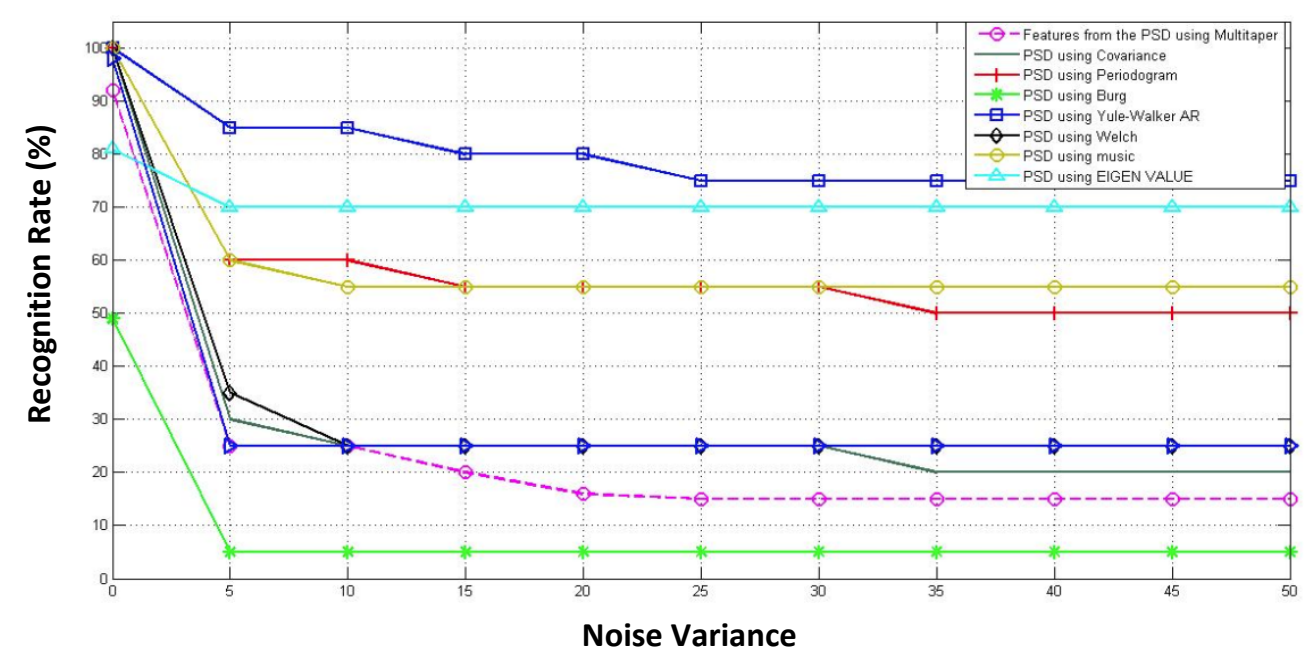

Fig. (17): Recognition rate versus noise variance for parametric, non-parametric and Eigen vector analysis with Gaussian, Rayleigh and Rician degradation 


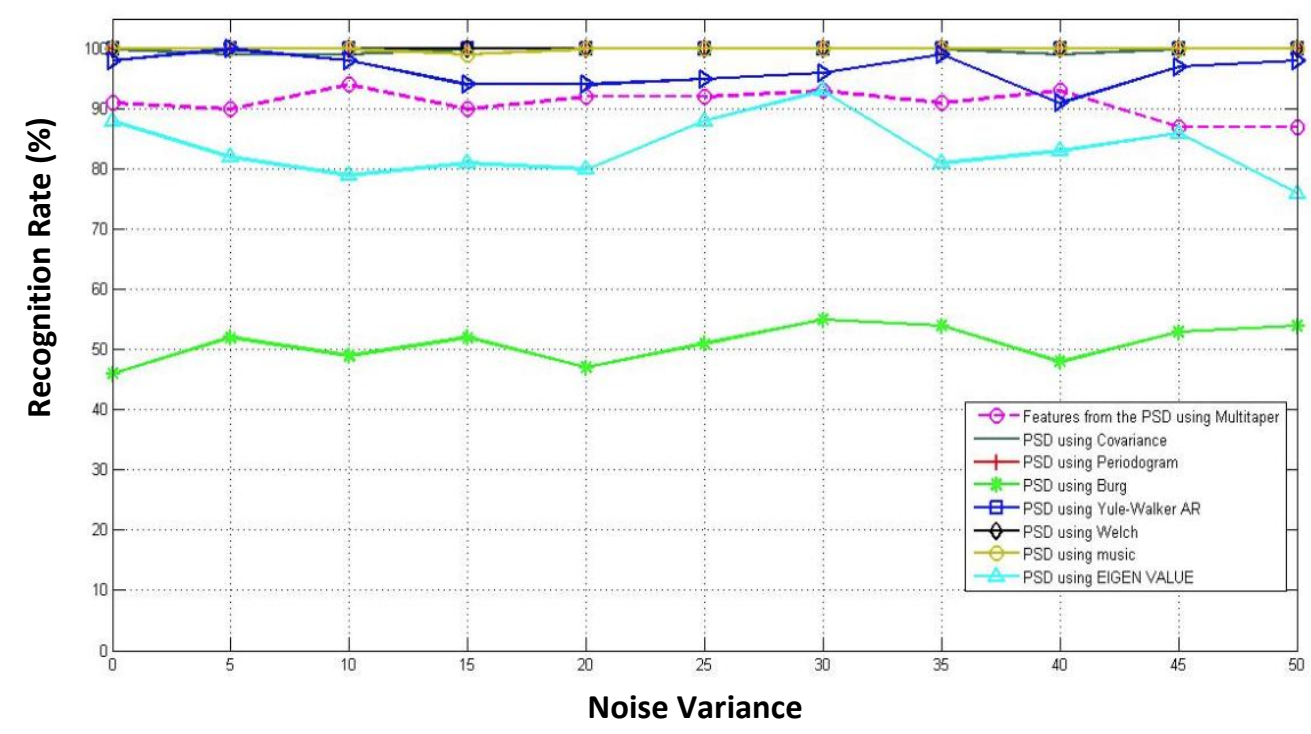

Fig. (18): Recognition rate versus noise variance for parametric, non-parametric and Eigen vector analysis with Salt pepper noise to the channel degradation

The recognition rate against the SNR at Gaussian noise degradation of the OFDMA signal is depicted in Fig. (11). From this Figure, the MUSIC method achieves the highest recognition rate compared to both parametric and non-parametric methods at the same SNR. However, the recognition rate against the noise variance at Rayleigh noise degradation of OFDMA signal is demonstrated in Fig. (12). The results show that the Welch, Yule-Walker and Periodogram methods have higher recognition rates at all values of noise variance. The recognition rate versus noise variance at Rician noise degradations is illustrated in Fig. (13). The obtained results confirm that the parametric method gives a higher recognition rate than other methods. Also, the extracted features from Burg methods introduce a lower recognition rate below $10 \%$ at moderate and higher noise variance.

Figure (14) shows the recognition rate versus noise variance for parametric, non-parametric and Eigenvector analysis with Gaussian \& Rayleigh degradation. As shown in this Figure, the features extracted from the Welch, Yule-Walker and Periodogram methods of the OFDMA signal give the highest recognition rate as compared to other methods. The recognition rate versus noise variance at Gaussian and Rician noise degradations is illustrated in Fig. (15). The obtained results confirm that Yule-Walker method introduces a higher recognition rate than other methods. However, the average recognition rate is around $80 \%$. Additionally, the recognition rate versus noise variance for parametric, non-parametric and Eigenvector analysis with Rayleigh and Rician degradation is depicted in Fig. (16). The results show that Yule-Walker method introduces a higher recognition rate than other methods. Furthermore, the average recognition rate is found to be $80 \%$. Figure (17) illustrates the recognition rate versus noise variance for parametric, non-parametric and Eigenvector analysis with Gaussian with Rayleigh and Rician degradation. From this Figure, the Yule-Walker has a higher recognition rate $(80 \%)$ than other methods. On the other hand, the features extraction of Burg methods achieves the lowest recognition rate at all values of noise variance. The variation of recognition rate versus noise variance for parametric, non-parametric and Eigenvector analysis with Salt \& pepper noise to the channel degradation is shown in Fig. (18). It was noted that Yule-Walker and MUSIC have a higher recognition rate of $100 \%$. The results in Figures(11-18) show that the recognition rate decreases with increasing of noise variance. Moreover, the above results illustrate that the features extracted from Yule-Walker parametric method achieve a higher recognition rate compared to other methods. However, the features extracted from the non-parametric method achieve the lowest recognition rate.

The features of the OFDMA signal are extracted from five HOS signals. These signals may be Bi-spectrum or cross Bi-spectrum signals. The first signal is based on the direct method of Bi-spectrum. The second one is the Bi-spectrum which is based on the indirect method. The third one is the Bi-spectrum and it is based on ARMA method. However, the fourth signal is the Bi-spectrum and it is based on Cumulates of ARMA method. Lastly, the cross Bi-spectrum signal is 
based on the direct method. In addition, 20 OFDMA signals are employed for training the various objects in the signal by using ANN. The ANN is used for training various objects in 20 OFDMA signals. Moreover, other 20 OFDMA signals are used for testing the OFDMA signal with various types of noise degradations. These degradations are Gaussian, Rayleigh, Rician, Gaussian with Rayleigh, Gaussian with Rician, Rayleigh with Rician, Gaussian with Rayleigh and Rician and Salt pepper.

The recognition rate against SNR at Gaussian degradation of OFDMA signal is depicted in Fig. (19). A high recognition rate is achieved with all methods except that extracted from the original signal. Moreover, the recognition rate against noise variance in the case of Rayleigh of OFDMA signal is illustrated in Fig. (20). From this Figure, the ARMA method gives a high recognition rate. For example, the recognition rate for ARMA model is $100 \%$ at a noise variance of 10 which is higher than the recognition rate of other methods at the same noise variance. It was observed that the recognition rate decreases by increasing the noise variance. Moreover, the recognition rate against noise variance in the case of degradations by Rician noise is shown in Fig. (21). The recognition rate of ARMA method is found to be $100 \%$ that is higher than that achieved by other methods at a noise variance of $15 \mathrm{~dB}$. Thus, the features extracted from the OFDMA signal based on ARMA model give the highest recognition rate in the case of Rician noise degradation.
The recognition rate versus the noise variance at noise degradations by Gaussian with Rayleigh is shown in Fig.(22). Also, the features extracted from HOS based on ARMA model achieved the best recognition rate. Furthermore, a recognition rate of $100 \%$ is obtained at all variations of noise variance. The recognition rate against the noise variance at noise degradation by Gaussian with Rician noise is illustrated in Fig. (23). The features extracted from the HOS based on ARMA method achieve the highest recognition rate. Figure (24) shows the recognition rate versus noise variance at Rayleigh with Rician channel noise degradation. From this Figure, the features extracted from ARMA method give the highest recognition rate at all variations of noise variance. The recognition rate against the noise variance at noise degradation by Gaussian with Rayleigh and Rician is depicted in Fig. (25). Similarly, the highest recognition rate of $100 \%$ is obtained with ARMA method for all values of noise variance.

Figure (26) illustrates the recognition rate versus noise variance at different Salt pepper noise of channel degradation. It was noted that the features extracted from the Bi-spectrum and ARMA methods introduce the highest recognition rate of $100 \%$. From the obtained results, the features extracted from the ARMA model achieve the highest recognition rate for all types of OFDMA signal noise degradations. Moreover, better results are obtained with ARMA models of HOS as compared to those of Yule-Walker models of the parametric PDF method.

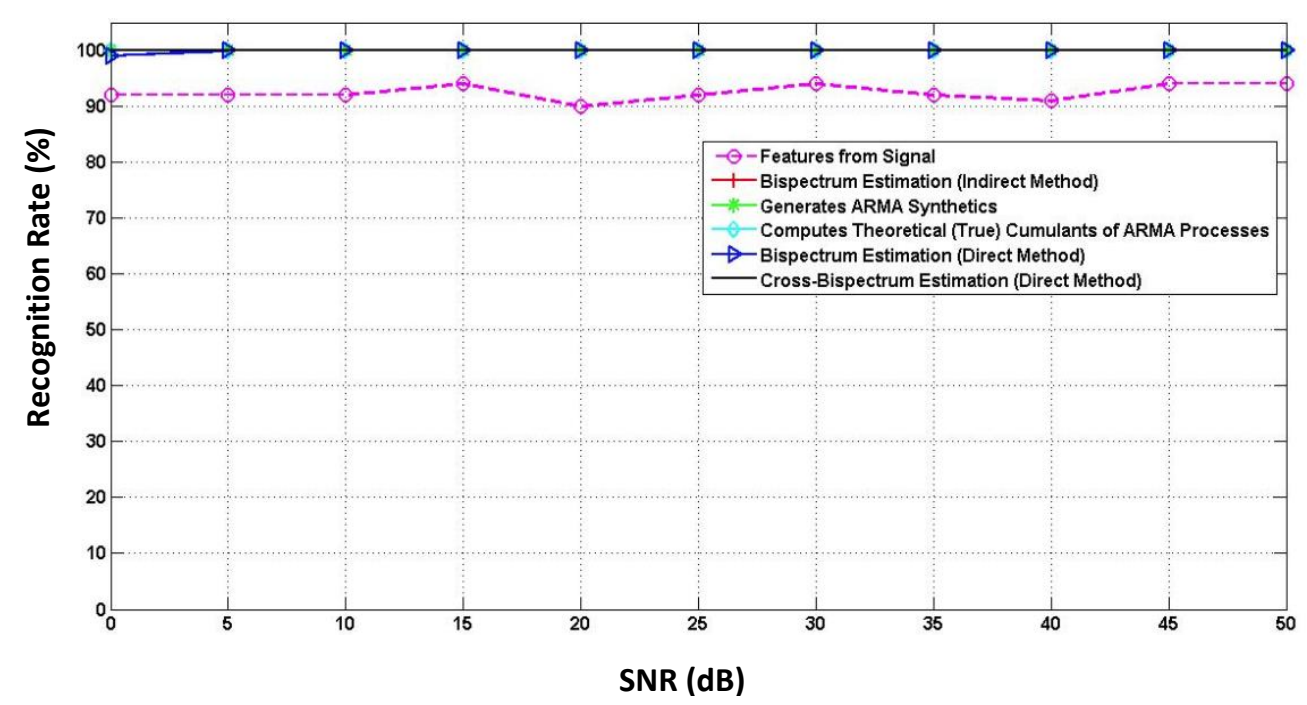

Fig. (19): OFDN signal recognition rate for bi-spectrum and cross bi-spectrum over Gaussian degradation 


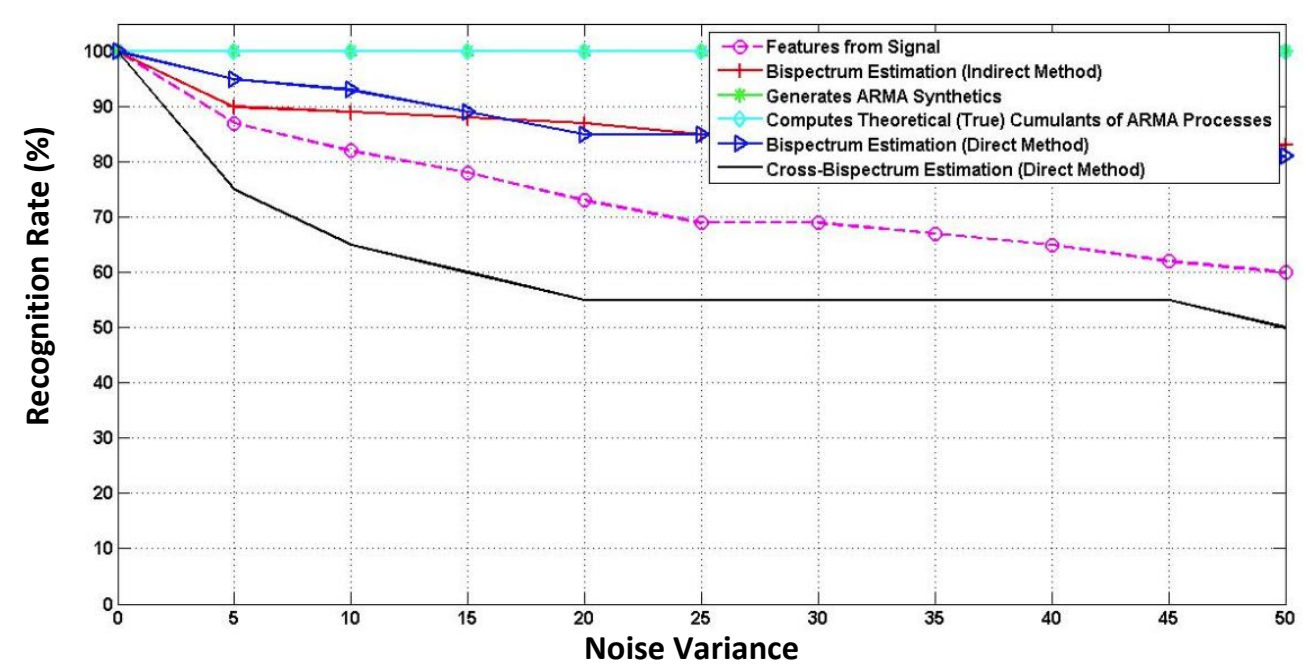

Fig. (20): OFDMA signal recognition rate for bi-spectrum and cross bi-spectrum with Rayleigh degradation

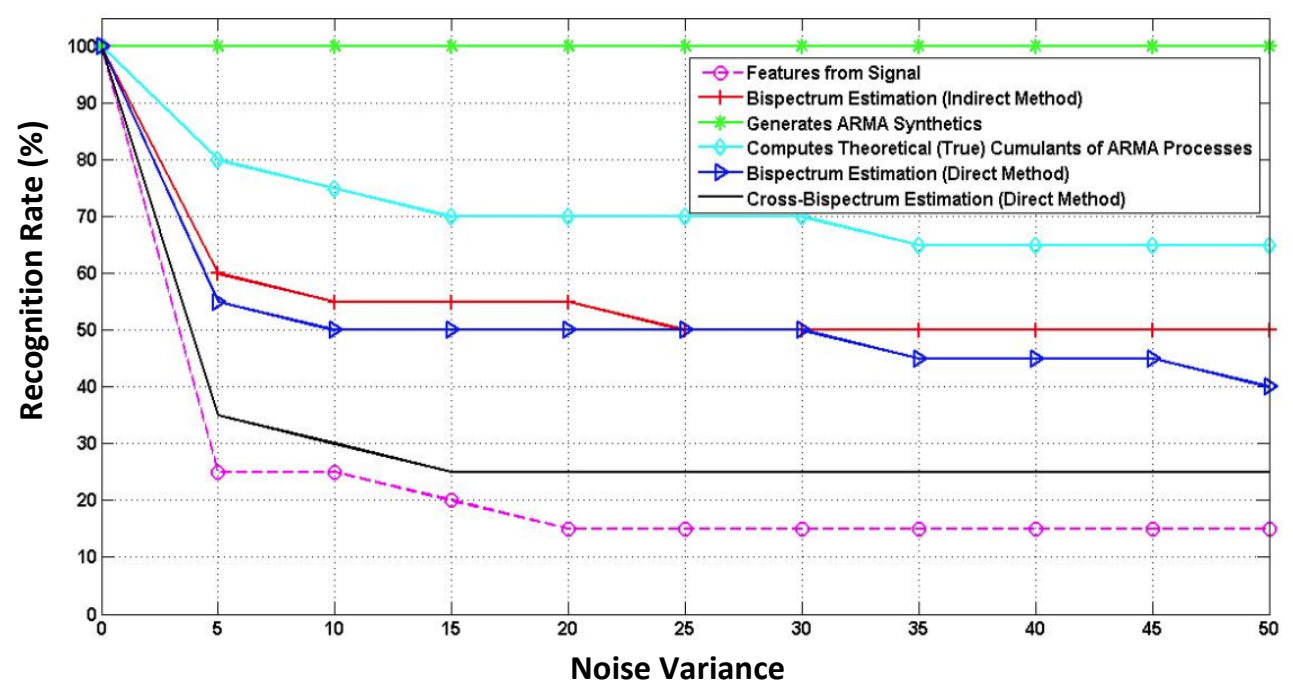

Fig. (21): OFDMA signal recognition for bi-spectrum and cross bi-spectrum with Rician degradation

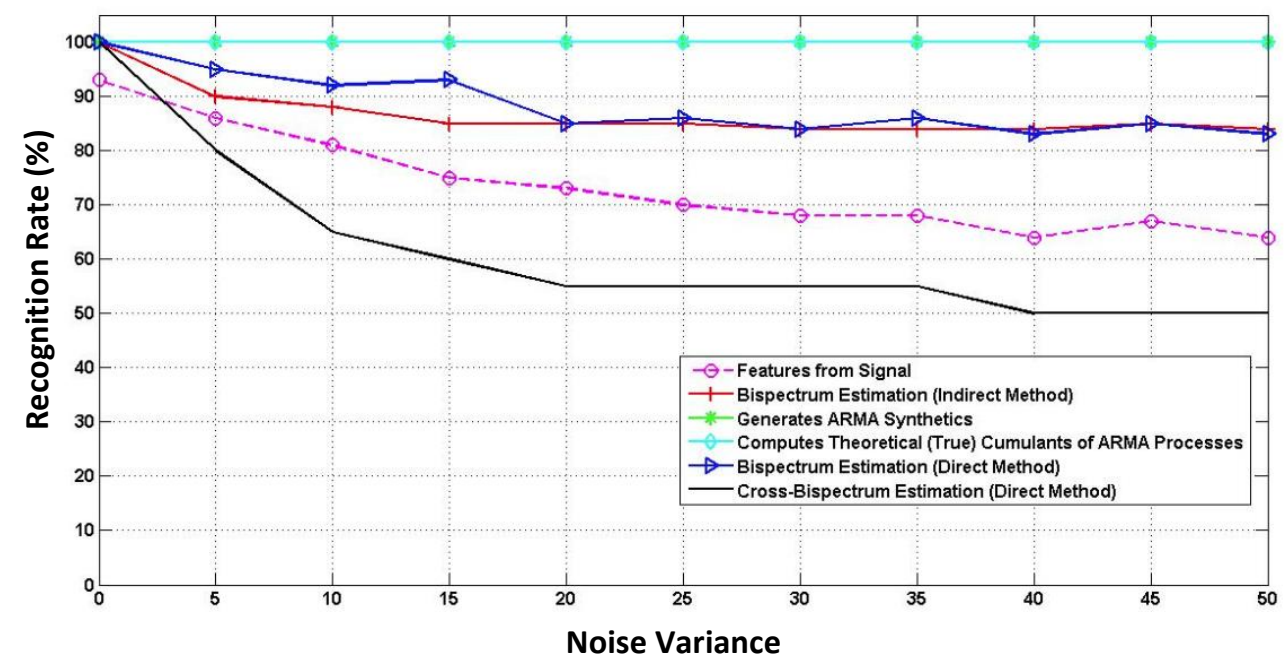

Fig. (22): OFDMA signal recognition rate for bi-spectrum and cross bi-spectrum with Gaussian and Rayleigh degradation 


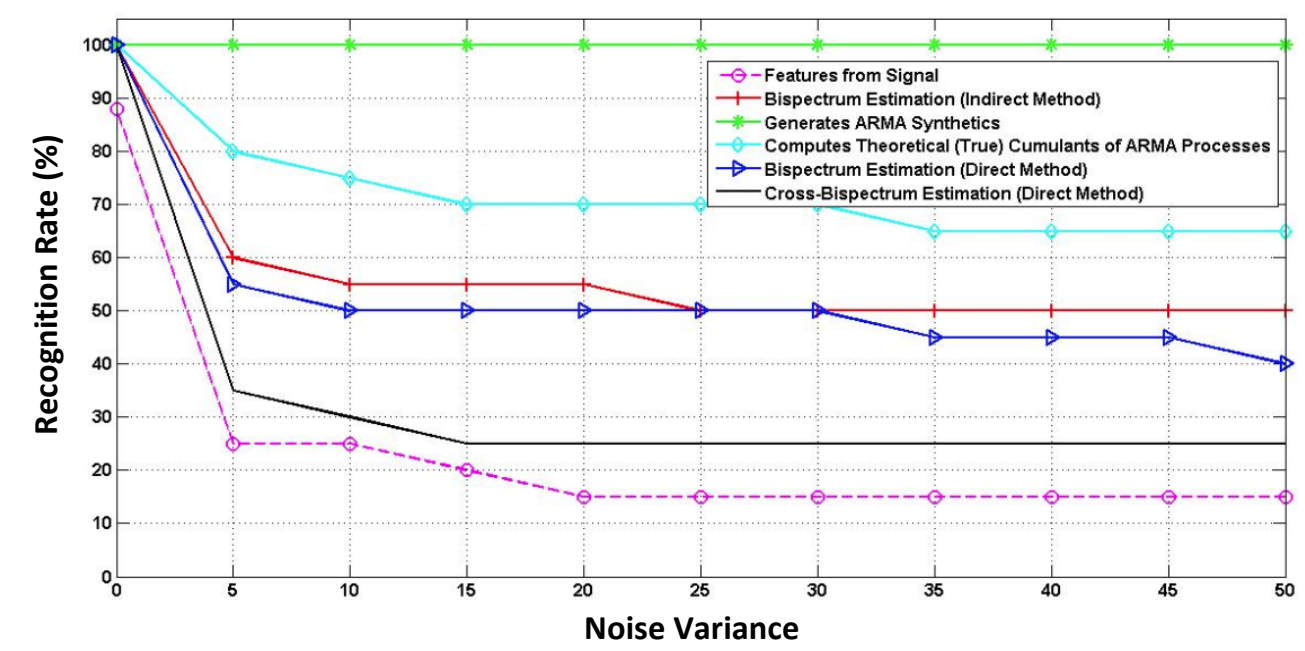

Fig. (23): OFDMA signals recognition for bi-spectrum and cross bi-spectrum with Gaussian and Rician degradation

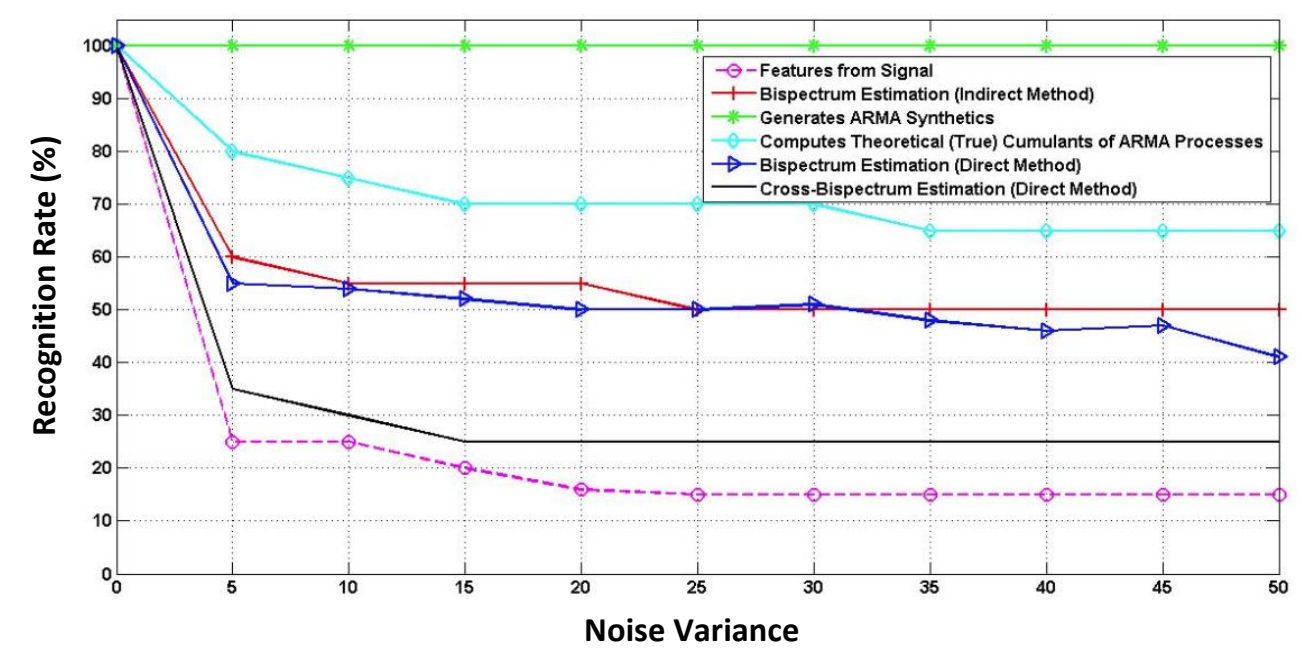

Fig. (24): OFDMA signals recognition for bi-spectrum and cross bi-spectrum with Rayleigh and Rician degradation

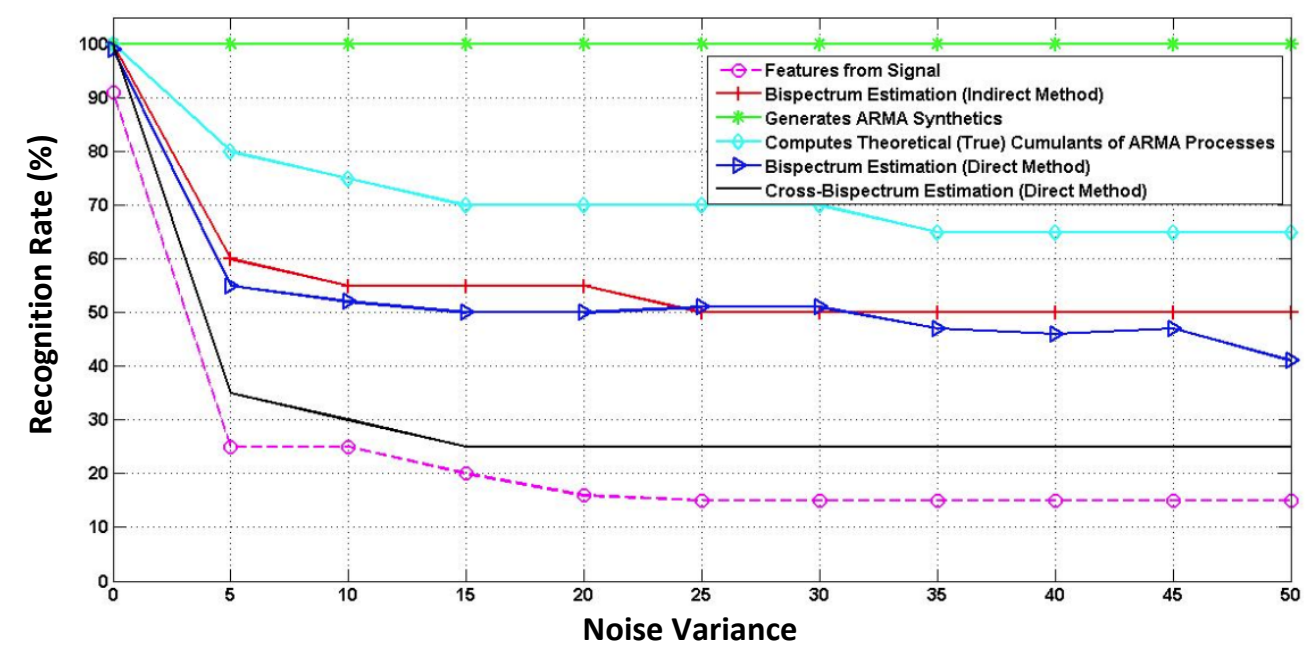

Fig. (25): OFDMA signals recognition for bi-spectrum and cross bi-spectrum with Gaussian, Rayleigh and Rician degradation 


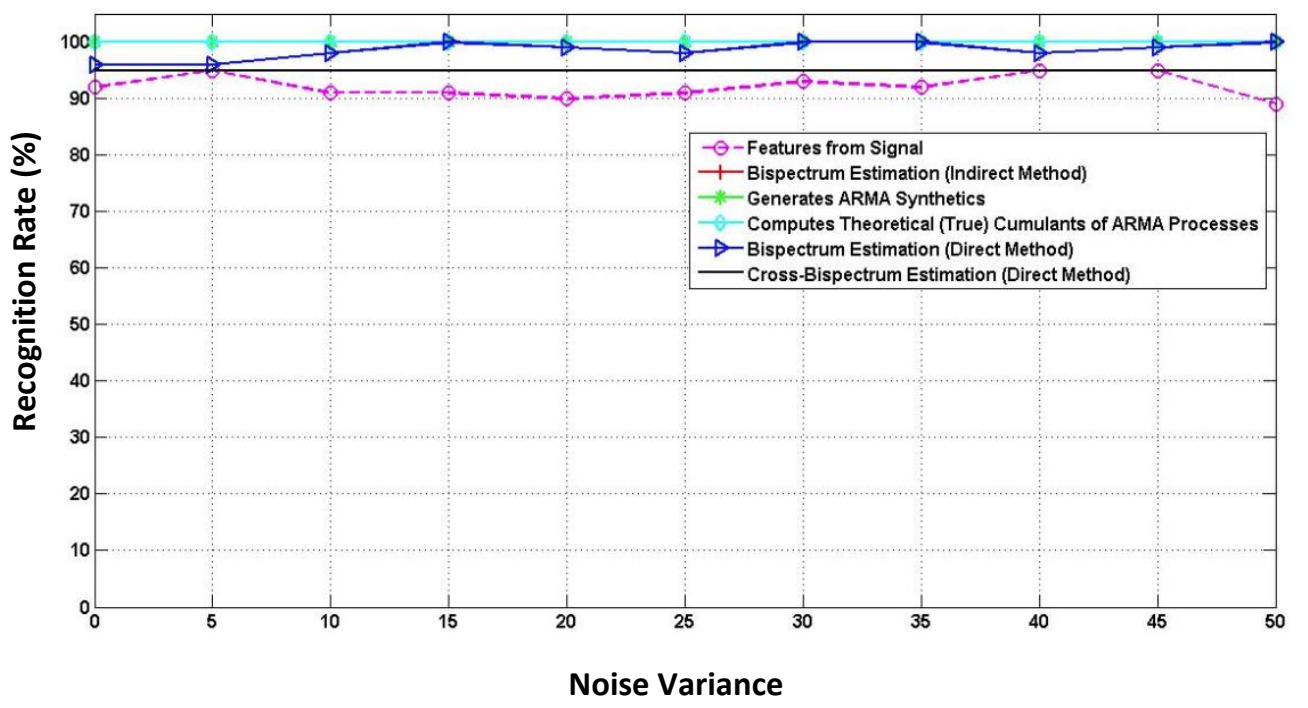

Fig. (26): OFDMA signals recognition for bi-spectrum and cross bi-spectrum with Salt \& pepper noise to the channel degradation

\section{CONCLUSION}

This manuscript is concerned with handling the high peak power issue within the OFDMA system. Moreover, the effect of multipath fading on the signal is analyzed through computational error rate. Recognition of this signal under channel fading is the scope of this research. Therefore, algorithms, for the reduction of high peak power and signal recovery, are introduced. These algorithms depend on the detection of maximum peaks of OFDMA signal from the high-power amplifier. These peaks are compared with the upper and lower threshold limits of the signal. It is noticed that the signal proposed recovery algorithm introduces efficient results based on statistical accuracy of maximum peaks. Moreover, the accuracy of the transmitted signal under various channel fading environments is proven. Rayleigh with Rician channel noise introduces the highest error rate. OFDMA signal identification through power density and order statistics algorithms is suggested. The features are extracted from both density spectrum and order statistics of the transmitted signal. It is observed that the order statistic method achieves the highest recognition rate of $100 \%$ at different values of SNR under different channel fading impairments. The results show that high order statistics introduce a higher recognition rate compared to the power spectrum of OFDMA signal under variation of noise attacks.

\section{CONFLICT OF INTEREST STATEMENT:}

There is no conflict of interest

\section{REFERENCES}

[1] Islam, G. Z. and Kashem, M. A. (2020) Efficient resource allocation in the IEEE 802.11ax network leveraging OFDMA technology, Journal of King Saud University - Computer and Information Sciences,

[2] P.S, S., P, M., and S.M, S. (2020) A low complexity iterative equalization technique for OFDMA and SC-FDMA uplink systems with TxRx IQ imbalance and CFO under doubly selective channels, Physical Communication, 42101170.

[3] Scanzio, S., Wisniewski, L., and Gaj, P. (2021) Heterogeneous and dependable networks in industry - A survey, Computers in Industry, 125 103388.

[4] Chen, C., Zhong, W.-D., and Wu, D. (2016) Color Multiplexing Based Unipolar OFDM for Indoor RGB LED Visible Light Communication, Procedia Engineering, 140 159-165.

[5] Jibreel, N., Elkawafi, S., Younis, A., and Mesleh, R. (2020) Performance analysis of sparse code multiple access with variant MIMO techniques, Physical Communication, 39101023.

[6] Kumar, A. and Kumar, K. (2020) Multiple access schemes for Cognitive Radio networks: A survey, Physical Communication, 38100953.

[7] Saini, R., Mishra, D., and De, S. (2019) Subcarrier pairing as channel gain tailoring: Joint resource allocation for relay-assisted secure OFDMA with 
untrusted users, Physical Communication, 32 217230.

[8] Vappangi, S. and Mani, V. V. (2019) Interference analysis and MUI-cancellation in DCO-OFDMAbased IM/DD systems for VLC, Optics Communications, 448 130-146.

[9] Yang, P., Shi, H., and Chen, X. (2016) EAM-based multiband OFDM systems incorporating PAPR reduction and SSII cancellation, Optics \& Laser Technology, 78 50-55.

[10] You, Y.-H., Jung, H.-K., and Jeon, W.-G. (2004) A Simple Peak-to-Average Power Ratio (PAR) Reduction Scheme for OFDM-CDMA Signals, $A E U$ - International Journal of Electronics and Communications, 58 (2), 142-146.

[11] Wang, Z. and Chen, S. (2015) Combined discrete Fourier transform precoding and clipping using direct detection optical OFDM, Optics Communications, 347 147-154.

[12] Wei-Ren, P., Kai-Ming, F., Willner, A. E., and Sien, C. (2009) Estimation of the Bit Error Rate for Direct-Detected OFDM Signals With Optically Preamplified Receivers, Journal of Lightwave Technology, 27 (10), 1340-1346.

[13] Xiao, Y., Chen, M., Li, F., Tang, J., Liu, Y., and Chen, L. (2015) PAPR reduction based on chaos combined with SLM technique in optical OFDM IM/DD system, Optical Fiber Technology, 21 8186.

[14] Wang, Y. and Tao, X.-f. (2007) Inter-antenna and subblock shifting and inversion for peak-to-average power ratio reduction in MIMO-OFDM systems, The Journal of China Universities of Posts and Telecommunications, 14 (4), 41-45.

[15] Abualigah, L. M., Khader, A. T., Hanandeh, E. S., and Gandomi, A. H. (2017) A novel hybridization strategy for krill herd algorithm applied to clustering techniques, Applied Soft Computing, 60 423-435.

[16] Abualigah, L. M., Khader, A. T., Al-Betar, M. A., and Alomari, O. A. (2017) Text feature selection with a robust weight scheme and dynamic dimension reduction to text document clustering, Expert Systems with Applications, 84 24-36.

[17] Bolaji, A. L. a., Al-Betar, M. A., Awadallah, M. A., Khader, A. T., and Abualigah, L. M. (2016) A comprehensive review: Krill Herd algorithm $(\mathrm{KH})$ and its applications, Applied Soft Computing, 49 437-446.

[18] Singh, A. and Singh, H. (2016) Peak to average power ratio reduction in OFDM system using hybrid technique, Optik, 127 (6), 3368-3371.

[19] Sung, M., Lee, J., and Jeong, J. (2013) Improvement in the nonlinear tolerance for a DFTspread CO-OFDM system using M-ASK modulation and Hermitian symmetry of a DFT, Optical Fiber Technology, 19 (6), 643-651.

[20] Ulovec, K., "Noncoherent recognition of OFDM modulation," presented at the 2008 18th International Conference Radioelektronika, 2008.

[21] Wu, W.-C. (2020) Toward the energy efficiency of resource allocation algorithms for OFDMA downlink MIMO systems, Journal of Electronic Science and Technology, 100007.

[22] Nadal, L., Svaluto Moreolo, M., Fàbrega, J. M., and Junyent, G. (2014) Low complexity PAPR reduction techniques for clipping and quantization noise mitigation in direct-detection O-OFDM systems, Optical Fiber Technology, 20 (3), 208216.

[23] Dong, Z., Cao, Z., Lu, J., Li, Y., Chen, L., and Wen, S. (2009) Transmission performance of optical OFDM signals with low peak-to-average power ratio by a phase modulator, Optics Communications, 282 (21), 4194-4197.

[24] Zhou, J., Zhang, Z., Zhang, T., Guo, M., Tang, X., Wang, Z., et al. (2016) A combined PAPRreduction technique for asymmetrically clipped optical OFDM system, Optics Communications, 366 451-456.

[25] Mayyala, Q., Abed-Meraim, K., and Zerguine, A. (2021) A class of multi-modulus blind deconvolution algorithms using hyperbolic and Givens rotations for MIMO systems, Signal Processing, 183107895.

[26] Kashany-Mizrahi, I. and Sadot, D. (2013) Low-cost adaptive directly modulated optical OFDM based on semiconductor optical amplifier, Optical Fiber Technology, 19 (5), 501-506.

[27] Abualigah, L. M., Khader, A. T., and Hanandeh, E. S. (2018) A combination of objective functions and hybrid Krill herd algorithm for text document clustering analysis, Engineering Applications of Artificial Intelligence, 73 111-125. 
[28] Abualigah, L. M., Khader, A. T., and Hanandeh, E. S. (2018) A new feature selection method to improve the document clustering using particle swarm optimization algorithm, Journal of Computational Science, 25 456-466.

[29] Abdullahi, S. U., Liu, J., and Mohadeskasaei, S. A. (2019) Efficient Resource Allocation with Improved Interference Mitigation in FFR-Aided OFDMA Heterogeneous Networksb, Journal of Electronic Science and Technology, 17 (1), 73-89.

[30] Hoang, T. M., Tan, N. T., Tran, X. N., and Dung, L. T. (2020) Performance analysis of power beaconassisted energy harvesting NOMA multi-user relaying system over Nakagami-m fading channels, $A E U$ - International Journal of Electronics and Communications, 115153022.

[31] Shin, C. and Park, H., "A Closed-Form Expression of Instantaneous Bit Error Rate for BIC-OFDM Systems," presented at the 2010 IEEE International Conference on Communications, 2010.

[32] Kaur, H., Kumar, M., Sharma, A. K., and Singh, H. P. (2016) Performance analysis of DWT based OFDM over fading environments for mobile WiMax, Optik, 127 (2), 544-547.

[33] Dahat, P. A. and Das, S. S. (2020) Device-to-device communications under transceiver impairments in OFDMA cellular networks, Physical Communication, 40101058.

[34] Oyerinde, O. O. (2018) An overview of channel estimation schemes based on regularized adaptive algorithms for OFDM-IDMA systems, Digital Signal Processing, 75 168-183.

[35] Olkkonen, H. and T, J., "Shift Invariant Discrete Wavelet Transforms," in Discrete Wavelet Transforms - Algorithms and Applications, ed: InTech, 2011.

[36] Hosseini, R. and Vafadust, M. (2008) Almost Perfect Reconstruction Filter Bank for, Journal of Wavelet Theory and Applications, 2 (1), 1-14.

[37] Dahat, P. A. and Das, S. S. (2015) QoS enhancement in OFDM based systems under transceiver impairments, Physical Communication, 16 25-36.

[38] Shukla, S., Shukla, S., and Purohit, N. (2013) PAPR Reduction in SC-FDMA Using NCT Technique, Procedia Technology, 10 927-934.
[39] Rahmatallah, Y., Bouaynaya, N., and Mohan, S., "Bit Error Rate Performance of Linear Companding Transforms for PAPR Reduction in OFDM Systems," presented at the 2011 IEEE Global Telecommunications Conference - GLOBECOM 2011, 2011.

[40] Hung, H.-L. (2011) Using evolutionary computation technique for trade-off between performance peak-to average power ration reduction and computational complexity in OFDM systems, Computers \& Electrical Engineering, 37 (1), 57-70.

[41] Siddiq, A. I. (2015) PAPR reduction in OFDM systems using peak insertion, $A E U$ - International Journal of Electronics and Communications, 69 (2), 573-578.

[42] Lee, B. M. and de Figueiredo, R. J. P. (2010) MIMO-OFDM PAPR reduction by selected mapping using side information power allocation, Digital Signal Processing, 20 (2), 462-471.

[43] Hmood, J. K., Noordin, K. A., Arof, H., and Harun, S. W. (2015) Peak-to-average power ratio reduction in all-optical orthogonal frequency division multiplexing system using rotated constellation approach, Optical Fiber Technology, 25 88-93.

[44] Nikias, C. L. and Raghuveer, M. R. (1987) Bispectrum estimation: A digital signal processing framework, Proceedings of the IEEE, 75 (7), 869891.

[45] Tsai, T.-Y., Tsai, Y.-H., Tsai, Z., and Sheu, S.-T. (2017) A Novel Description approach based on sorted rectangles for scheduling information bearing in OFDMA systems, Computer Networks, 115 82-99.

[46] Zahran, O., Kasban, H., Abd El-Samie, F. E., and El-Kordy, M. (2012) Power density spectrum for the identification of residence time distribution signals, Applied Radiation and Isotopes, 70 (11), 2638-2645.

[47] Liu, F., Yang, Q., He, Q., and Kwak, K. S. (2018) Energy efficiency and spectral efficiency tradeoff in downlink OFDMA systems with imperfect CSI, $A E U$ - International Journal of Electronics and Communications, 85 54-58.

[48] Olabiyi, O. and Annamalai, A., "Analysis and new implementations of periodogram-based spectrum sensing," presented at the 2012 35th IEEE Sarnoff Symposium, 2012. 
[49] Alkan, A. and Yilmaz, A. S. (2007) Frequency domain analysis of power system transients using Welch and Yule-Walker AR methods, Energy Conversion and Management, 48 (7), 2129-2135.

[50] Kasban, H., Arafa, H., Elaraby, S. M., Zahran, O., and El-Kordy, M., "RTD signal identification using linear and nonlinear modified periodograms," presented at the The 2011 International Conference on Computer Engineering \& Systems, 2011.

[51] Kasban, H., "Applying advanced digital signal processing techniques in industrial radioisotopes applications," PhD, Electrical Communication, Menofia Egypt, 2012.

[52] Mendel, J. M. (1991) Tutorial on higher-order statistics (spectra) in signal processing and system theory: theoretical results and some applications, Proceedings of the IEEE, 79 (3), 278-305.

[53] Kasban, H. (2016) Fingerprints verification based on their spectrum, Neurocomputing, 171 910-920.

[54] Kasban, H. and Hamid, A. (2014) Spectrum analysis of radiotracer residence time distribution for industrial and environmental applications, Journal of Radioanalytical and Nuclear Chemistry, 300 (1), 379-384.

[55] Lin, B., Tang, X., Li, Y., Zhang, M., Lin, C., and Ghassemlooy, Z. (2017) Experimental demonstration of IDMA-OFDM for passive optical network, Optics Communications, 403 222-225.

[56] Baştürk, İ. and Özbek, B. (2016) Radio resource management for user-relay assisted OFDMA-based wireless networks, AEU - International Journal of Electronics and Communications, 70 (5), 643-651.

[57] Wu, W.-C. and Hsu, C.-Y. (2017) Subcarriers-andits allocation algorithms for downlink OFDMAbased MIMO systems, Journal of the Franklin Institute, 354 (11), 4615-4636.

[58] Luise, M., Reggiannini, R., and Vitetta, G. M. (1998) Blind equalization/detection for OFDM signals over frequency-selective channels, IEEE Journal on Selected Areas in Communications, 16 (8), 1568-1578.

[59] Mahmoud, I. I., Elgazzar, O. H., Hashima, S., and Konber, H. A. (2018) Analysis and performance evaluation of Relay Assisted OFDMA cellular systems with different Beamforming techniques, Physical Communication, 31 49-61.

[60] Nasralla, M. M., Khan, N., and Martini, M. G. (2018) Content-aware downlink scheduling for LTE wireless systems: A survey and performance comparison of key approaches, Computer Communications, 130 78-100. 